\title{
REVIEW
}

Open Access

\section{Implementing exercise programs to prevent falls: systematic descriptive review}

\author{
Victoria Shier $^{1 *}$ (D), Eric Trieu ${ }^{2}$ and David A. Ganz ${ }^{3}$
}

\begin{abstract}
Background: The United States Preventive Services Task Force recommends exercise to prevent falls in community-dwelling adults aged $\geq 65$ years at increased fall risk. However, little is known about how best to implement exercise programs in routine care when a patient's need for exercise is identified within the healthcare system.

Methods: Using a qualitative approach, we reviewed the literature to determine how exercise programs to prevent falls are implemented from the vantage point of a health care setting. We synthesized descriptive information about each program with data on program features and implementation difficulties and facilitators.

Results: We found that programs sponsored by primary care providers (PCPs) or specialists may help with recruitment into exercise programs. PCPs have the opportunity to identify people at risk and promote participation since most older adults regularly visit, and inquire about exercise from, their physicians. In terms of referral options, both home-based and group-based exercise programs have been shown effective in preventing falls; however, each approach carries strengths and limitations. Home-based programs can include participants who are reluctant or unable to attend group classes and can be individually tailored, but provide less opportunity for supervision and socialization than classes. Adherence to programs can be encouraged, and attrition minimized, through positive reinforcement. Successful programs ranged in expense for exercise sessions: a weekly class combined with exercises at home cost $<\$ 2$ per participant per week, while frequent individual sessions cost $>\$ 100$ per participant per week.

Conclusions: With increasing attention to population-based health management in the United States, clinicians and health system leaders need a deeper understanding of how to link patients in their healthcare systems with appropriate community programs. This review identifies key characteristics of successful fall prevention exercise programs that can be used to determine which local options conform to clinical evidence. In addition, we highlight tradeoffs between program options, such as home versus group exercise programs, to allow referrals to be tailored to local conditions and patient preferences. Finally, our work highlights the key role of the PCP in recruiting patients to participate in exercise programs, and identifies options, such as registries, to support referrals to the community.
\end{abstract}

Keywords: Fall prevention, Exercise program, Descriptive systematic review

\footnotetext{
*Correspondence: vshier@prgs.edu

${ }^{1}$ Pardee RAND Graduate School, RAND Corporation, 1776 Main St, Santa

Monica, CA 90407, USA

Full list of author information is available at the end of the article
} 


\section{Review}

\section{Background}

More than one-third of adults age $\geq 65$ fall each year (Rubenstein and Josephson 2002; Tinetti 2003). Many people who fall suffer injuries such as lacerations, hip fractures, and head trauma (Stevens et al. 2006a) and may experience reduced mobility and physical fitness. Falls are the leading cause of injury-related deaths among adults age $\geq 65$ (Centers for Disease Control and Prevention, 2016). The resulting hospitalizations, emergency department visits, and other treatments from falls are costly. Adjusted for inflation the annual direct medical cost of falls in the U.S. has been estimated at $\$ 34$ billion (Centers for Disease Control and Prevention, 2016, Stevens et al. 2006b).

Several systematic reviews have demonstrated that exercise programs are effective in reducing falls and fallrelated injuries in community-dwelling elders (El-Khoury et al. 2013; Gillespie et al. 2012; Michael et al. 2010; Sherrington et al. 2011; Sherrington et al. 2008). In addition, based on its own review of the evidence, the United States Preventive Services Task Force (USPSTF) recommended exercise or physical therapy as a primary care-relevant intervention to prevent falls in communitydwelling adults age $\geq 65$ who are at increased risk for falls (Moyer 2012). However, despite the strong evidence base supporting the effectiveness of exercise programs to prevent falls, surprisingly little is known about how best to implement an exercise program when a patient's need for exercise is identified in a health care setting, as noted in a commentary by Tinetti and Brach (2012). Such information is needed by primary care providers interested in implementing or referring patients to exercise-based fall prevention programs and to inform potential coverage determinations and implementation strategies by the Centers for Medicare and Medicaid Services (CMS) and commercial health plans. We hypothesized that although the current evidence base is organized around effectiveness, we could glean critical information to guide implementation of exercise programs using a qualitative approach to literature review (Centre for Reviews and Dissemination 2009). In this article we detail our findings from the review, and then focus on the implications for integration of exercise programs for prevention of falls with existing healthcare delivery systems.

\section{Methods}

Seven relevant and recent systematic reviews of fall prevention interventions (Beswick et al. 2008; El-Khoury et al. 2013; Gates et al. 2008; Gillespie et al. 2012; Michael et al. 2010; Sherrington et al. 2011; Sherrington et al. 2008) served as source material for our analysis. All potential studies included in these systematic reviews, hereafter referred to as source articles, were assessed by two reviewers to determine whether they met the following criteria: (1) enrolled independently-living adults of mean age $\geq 65$ years; (2) structured exercise or physical therapy was the only or core component of the intervention (all treatment participants needed to receive the exercise component for exercise to be a core component); (3) participants were recruited from, or the program was administered from a health care setting (defined as a location where a trained healthcare professional practices either on site or in the vicinity); (4) study design was a randomized controlled trial (RCT) and control group did not receive exercise; (5) study reported number of falls over a given period of time, number of people who fell over a given period, or time to first fall, and (6) study was reported in English. We limited our search to RCTs to focus on data derived from the same studies that have informed current guidelines. Differences regarding inclusion/exclusion of articles were resolved by consensus.

To augment the data provided in the original studies, we searched for related articles by reviewing source article bibliographies and searching for articles citing the source article in Web of Science and Google Scholar. The goal of searching for related articles was to find process evaluations that documented and evaluated implementation steps in detail.

Data were extracted by two reviewers using a standardized form. Discrepancies were resolved by discussion, with involvement of a third reviewer if necessary. The studies were described in terms of patient fall risk (above-average versus average/below-average), presence of exercise components using the taxonomy developed by Prevention of Falls Network Europe project (Lamb et al. 2011), aspects of the exercise program (setting, amount of supervision, progression of exercises, intensity of exercise), and intervention success. The study sample was classified as at above-average risk for falls if the baseline annualized probability of at least one fall was higher than $36 \%$ in the sample, which is the upper limit of the $95 \%$ confidence interval for the probability of falling at least once in any given year for an unselected sample of individuals age $\geq 65$ (Ganz et al. 2007). If baseline data were not presented, we used the annualized probability of at least one fall among the control participants during the study period. We classified studies as successful if the pooled estimate of the effect of exercise on the rate of falls was below 0.91 (the upper limit of the $95 \%$ confidence interval of the pooled estimate of the effect in a recent meta-analysis of exercise interventions; pooled rate ratio 0.84, $95 \%$ CI 0.77-0.91; (Sherrington et al. 2011)). We estimated costs for exercise instruction per participant per week by multiplying the time of supervised exercise per week by the Bureau of Labor Statistics 
mean wage estimate in 2015 U.S. dollars for the instructor's occupation (US Department of Labor, 2016). Quality of the RCTs (Table 2) was assessed by examining the Physiotherapy Evidence Database (PEDro) scale (www.pedro.org.au) which assesses the RCT on 10 criteria including random allocation, concealed allocation, and blinding of subjects, therapists, and assessors.

The research team identified the main categories important for implementation (e.g., recruitment, participant adherence, participant attitudes, implementation successes, difficulties and facilitators) and extracted the relevant qualitative data. This qualitative data was further coded using software (MAXQDA 10; Berlin, Germany) to identify themes within these categories.

\section{Results}

\section{Study characteristics}

Of the 128 relevant studies included in the seven systematic reviews, 29 met inclusion criteria. Seven were included in El-Khoury et al. 2013; 24 were included in Gillespie et al. 2012; 12 were included in Michael et al. 2010; 17 were included in Sherrington et al. 2008; 22 were included in Sherrington et al. 2011; and 1 came from our personal files. 22 of these were included in multiple systematic reviews. An additional 32 related articles that provided contextual information on the included RCTs were identified. Studies were excluded for the following reasons: 1 was not published in English, 41 studies did not include exercise or physical therapy as a core component, 3 were not RCTs, 1 study's control group received exercise, 19 did not include independently-living adults with a mean age $\geq 65$ years, 4 did not report falls outcome data, and 30 did not derive from a health care setting.

Studies took place in the following countries: Australia, Brazil, Canada, Chile, Finland, Germany, Japan, the Netherlands, Sweden, Switzerland, UK, and US. Table 1 provides a description of the participants, type of exercise, exercise setting, and instructors for successful and unsuccessful studies. Twenty studies were successful; nine were unsuccessful. Most included studies focused on a population at above-average risk for falls including patients with Parkinson's disease or recent history of injurious falls. Physical therapists provided exercise instruction and supervision in a majority of programs (Ashburn et al. 2007a; Campbell et al. 1999b; Campbell et al. 1997; Campbell et al. 2005; Clemson et al. 2012; Green et al. 2002; Haines et al. 2009; Hornbrook et al. 1994; Kronhed et al. 2009; Latham et al. 2003a; Lin et al. 2007; Luukinen et al. 2007; Madureira et al. 2007; Protas 2005; Steadman et al. 2003; Swanenburg et al. 2007). Tai chi or exercise instructors provided instruction in three studies (Barnett et al. 2003; Dangour et al. 2011; Li et al. 2005; Logghe et al. 2009) and nurses supervised in two studies (Ebrahim et al. 1997; Robertson et al. 2001).
Table 1 Study characteristics

\begin{tabular}{ll}
\hline & All studies $(n=29)$ \\
\hline Population & \\
Average age of participants (years) & 74 \\
\% women & $66 \%$ \\
Above-average risk population, $n(\%)^{a}$ & $22(76 \%)$ \\
Type of exercise & \\
Gait, Balance, and functional training, $n$ (\%) & $21(72 \%)$ \\
Strength/resistance, $n$ (\%) & $21(72 \%)$ \\
General physical activity, $n(\%)$ & $12(41 \%)$ \\
Flexibility, $n$ (\%) & $8(28 \%)$ \\
Endurance, $n$ (\%) & $6(21 \%)$ \\
3D (Tai Chi, Qi Gong, Dance), $n(\%)$ & $3(10 \%)$ \\
Other, $n$ (\%) & $1(3 \%)$ \\
Setting & \\
Home, $n$ (\%) & $9(31 \%)$ \\
Class, $n$ (\%) & $8(28 \%)$ \\
Class and home, $n(\%)$ & $5(17 \%)$ \\
Clinic, hospital, other, $n(\%)$ & $7(24 \%)$ \\
Instructor & $7(24 \%)$ \\
Physical therapists, $n$ (\%) & $16(55 \%)$ \\
Tai chi or exercise instructor, $n(\%)$ & $4(14 \%)$ \\
Nurse, $n(\%)$ & $2(7 \%)$ \\
Other, $n$ (\%) & \\
\hline
\end{tabular}

${ }^{a}$ Above-average risk population: Study sample classified as at above-average risk for falls if baseline annualized probability of at least one fall was higher than $36 \%$ in the sample. This is the upper limit of the $95 \%$ confidence interval for the probability of falling at least once in any given year for an unselected sample of individuals age $\geq 65$ (Ganz et al. 2007)

We have organized key lessons about exercise programs into two major categories: program design and implementation (patient population; recruitment; type of exercise; tailoring to individuals; location; supervision; exercise frequency and program duration; and participant adherence during the program), and sustainability and scalability (program attrition; maintaining participant exercise after intervention; and resource implications).

\section{Program design and implementation Patient population}

For older adults identified through a healthcare setting, authors of several studies suggested that frail older people have the most to gain from interventions to reduce falls (Campbell et al. 1997; Carter et al. 2002; Robertson et al. 2001). For example, a meta-analysis of studies implementing the Otago Exercise Program, a home-based program of balance and strength training and walking, found the program is "significantly more effective in reducing the rate of fall injuries in those aged 80 and older than in younger trial participants... The program may be most 
effective in frailer, older people because the exercises increase strength and balance above the critical threshold needed for activities such as rising from a chair and going up and down the stairs and over home hazards (Robertson et al. 2002)." However, authors of studies also suggested that programs may experience less withdrawal among healthier patients (Logghe et al. 2011) and changing behaviors of very frail patients may be challenging (Ashburn et al. 2007a). While not focused on patients in the health care setting, a recent review found no difference in the effectiveness of exercise interventions delivered to the general community and to individuals with elevated risk (Gillespie et al. 2012).

\section{Recruitment}

The most common recruitment strategy included an initial letter from primary care providers (PCPs) (Table 2). Authors of three studies reported that having a program sponsored by the primary care practice or specialist may help with recruitment (Ashburn et al. 2007a; Barnett et al. 2003; Campbell et al. 1997). PCPs have the opportunity to identify people at risk and promote participation since most older adults visit their PCP at least once each year, seek advice about exercise from their physicians, and are more likely to participate in exercise if recommended by their physician (Barnett et al. 2003). However, as discussed in some studies, the success of these recruitment efforts may depend on the motivation and enthusiasm of the health professionals (Ashburn et al. 2007b; Campbell et al. 1997). Another potential recruitment method was the "use of similarly aged persons for personal contact with participants... [this] appealed to the credibility and sense of ownership for the participants (Stevens et al. 1992)."

Within people age $\geq 65$, there were unique challenges to recruiting both younger and older subgroups. The youngest older adults were more likely to be busy with work or travel and the oldest were more likely to be ineligible because of physical limitations, medical problems, or institutionalization. Authors of studies reported that involving family members and PCPs in recruitment (Campbell et al. 1997) and offering free transportation might be particularly important for frail older adults (Stevens et al. 1992).

\section{Type of exercise}

The type of exercise in successful programs varied substantially (Table 2). Gait, balance, and functional training and strength/resistance training were the most common. Programs also targeted activities such as flexibility, general physical activity, endurance, and 3D exercise (Tai Chi, dance). Only five studies involved single components of exercise (Ebrahim et al. 1997; Li et al. 2005; Logghe et al.
2009; Luukinen et al. 2007; Steadman et al. 2003) and only one of these was successful (Li et al. 2005).

\section{Tailoring program to individuals}

Programs ranged from completely standardized to completely individualized, with intermediate strategies such as having standardized weight-bearing exercises with the progression to heavier weights tailored to each individual. One study tailored the program for each individual by suggesting strategies to improve balance that can be integrated into the participant's everyday activities (Clemson et al. 2012). Authors of another study noted that, particularly among frail participants, "The program needs to be individually tailored because older people vary considerably in their physical capacity and health in response to exercise (Gardner et al. 2001)."

\section{Location}

Studies that included participants at above-average risk implemented exercise programs in a range of settings. Home-based programs typically involved the instructor making an initial visit to the participant's home, followed by additional visits to increase the difficulty of the exercise or reinforce adherence. Exercise programs in the participant's home had the advantage that they could be followed indefinitely (Campbell et al. 2002) and may be easier to maintain than group programs (Campbell et al. 1997). However, home-based programs offer less supervision (Haines et al. 2009). Authors of one study noted that the "use of highintensity exercise in a home-based program might have had a greater risk of injuries and adverse events in these participants than a program of lower intensity...Most highintensity programs for older people have been conducted under highly supervised conditions (Latham et al. 2003a)."

Exercise programs delivered in class settings have several advantages including social interaction (Carter et al. 2002; Hornbrook et al. 1994; Logghe et al. 2009), peer reinforcement and encouragement (Carter et al. 2002; Hauer et al. 2001; Hornbrook et al. 1994), and efficient use of instructor time (Carter et al. 2002; Hornbrook et al. 1994). However, some participants do not want to attend group exercise sessions, and illness may leave a participant unable to resume the exercises at the class level (Campbell and Robertson 2010).

Studies that combined class and home components included a class program with homework assignments or instructions for continuing exercises at home after the class ended.

\section{Supervision}

Most home-based programs used a combination of home visits and telephone calls to supervise participants. Instructors made weekly (Haines et al. 2009), monthly (Ashburn et al. 2007a; Ebrahim et al. 1997), or 
Table 2 Summary of exercise programs for included studies

\begin{tabular}{|c|c|c|c|c|c|c|c|c|}
\hline $\begin{array}{l}\text { Authors (year) } \\
\text { Successful/ } \\
\text { Unsuccessful }^{a} \\
\text { Quality Score } \\
\text { (of 10) }^{b}\end{array}$ & $\begin{array}{l}\text { Exercise setting; } \\
\text { Standardized (S), } \\
\text { Individualized (I), } \\
\text { Semi- } \\
\text { individualized (SI) }\end{array}$ & $\begin{array}{l}\text { Population } \\
\text { and } \\
\text { recruitment }\end{array}$ & $\begin{array}{l}\text { Above-average } \\
\text { risk or Average/ } \\
\text { below-average } \\
\text { risk }^{c}\end{array}$ & $\begin{array}{l}\text { Exercise description } \\
\text { and Instructor }\end{array}$ & $\begin{array}{l}\text { Supervised/ } \\
\text { Unsupervised: Exercise } \\
\text { components } \\
\text { Time, Frequency and } \\
\text { Program Duration }\end{array}$ & Adherence & $\begin{array}{l}\text { Attrition } \\
\text { rate }\end{array}$ & $\begin{array}{l}\text { Exercise } \\
\text { instruction } \\
\text { cost per } \\
\text { participant }\end{array}$ \\
\hline $\begin{array}{l}\text { Ashburn et al. } \\
\text { (2007a) } \\
\text { Successful } \\
\text { (Albanese 2007; } \\
\text { Ashburn 2007b; } \\
\text { Ashburn 2008) } 8\end{array}$ & Home; I & $\begin{array}{l}\text { Patients with } \\
\text { idiopathic } \\
\text { Parkinson's } \\
\text { disease with } \\
\text { more than } 1 \\
\text { fall in past } \\
\text { year identified } \\
\text { through clinical } \\
\text { registers of } \\
\text { specialists; } \\
\text { letter sent }\end{array}$ & $\begin{array}{l}\text { Above-average } \\
\text { Risk }\end{array}$ & $\begin{array}{l}\text { Treatment goals established } \\
\text { and exercises from exercise } \\
\text { menu were taught in } \\
\text { home. Menu designed with } \\
6 \text { levels of progression } \\
\text { and comprised muscle } \\
\text { strengthening, range } \\
\text { of movement, balance } \\
\text { training and walking. } \\
\text { Exercises chosen } \\
\text { at appropriate level and } \\
\text { progressed at each visit. } \\
\text { Patients encouraged to } \\
\text { continue exercise after } \\
6 \text { weeks. } \\
\text { Instructor: physiotherapist }\end{array}$ & $\begin{array}{l}\text { Supervised: Balance, } \\
\text { Strength, Flexibility, } \\
\text { General physical } \\
\text { activity } \\
\text { - } 1 \text { hr, } 1 \text { time per week, } \\
6 \text { weeks } \\
\text { Unsupervised: Balance, } \\
\text { Strength, Flexibility, } \\
\text { General physical activity } \\
\text { - Daily }\end{array}$ & $\begin{array}{l}69 \text { of } 70 \text { had at } \\
\text { least } 6 \text { sessions }\end{array}$ & $\begin{array}{l}2 \% \text { per } \\
\text { month }\end{array}$ & $\begin{array}{l}\$ 41 \text { per } \\
\text { week }\end{array}$ \\
\hline $\begin{array}{l}\text { Barnett et al. } \\
(2003) \\
\text { Successful } 8\end{array}$ & $\begin{array}{l}\text { Class and } \\
\text { Home; S }\end{array}$ & $\begin{array}{l}\text { Women and men } \\
\text { aged } 65 \text { years } \\
\text { and older } \\
\text { recruited } \\
\text { from general } \\
\text { practice clinics } \\
\text { or acute hospital } \\
\text { physiotherapy } \\
\text { departments }\end{array}$ & $\begin{array}{l}\text { Above-average } \\
\text { Risk }\end{array}$ & $\begin{array}{l}\text { Group exercise class to improve } \\
\text { balance, coordination, aerobic } \\
\text { capacity and muscle strength. } \\
\text { Participants also received a home } \\
\text { exercise program based on } \\
\text { class content. } \\
\text { Also received information on } \\
\text { practical strategies for avoiding falls. } \\
\text { Instructor: accredited exercise } \\
\text { instructor }\end{array}$ & $\begin{array}{l}\text { Supervised: Strength, } \\
\text { Balance, Endurance, } \\
\text { Flexibility, 3D } \\
\text { - } 1 \text { hr, } 1 \text { time per } \\
\text { week, } 1 \text { year } \\
\text { Unsupervised: based } \\
\text { on class content but } \\
\text { not specified }\end{array}$ & $\begin{array}{l}\text { Participants } \\
\text { attended median } \\
\text { of } 23 \text { classes; } \\
91 \% \text { of those who } \\
\text { attended classes } \\
\text { performed } \\
\text { home exercises } \\
1+\text { times per week }\end{array}$ & $\begin{array}{l}2 \% \text { per } \\
\text { month }\end{array}$ & $\begin{array}{l}\$ 2 \text { per } \\
\text { week }\end{array}$ \\
\hline $\begin{array}{l}\text { Buchner et al. } \\
\text { (1997) } \\
\text { Successful } \\
\text { (Buchner et al. } \\
\text { 1993a; } \\
\text { Buchner 1993b; } \\
\text { Ory et al. 1993) } 7\end{array}$ & Class; S & $\begin{array}{l}\text { Women and men } \\
\text { aged } 68 \text { to } 85 \\
\text { years old with } \\
\text { mild deficits in } \\
\text { strength and } \\
\text { balance enrolled } \\
\text { in HMO; PCP } \\
\text { approved } \\
\text { participation } \\
\text { and sent letter }\end{array}$ & $\begin{array}{l}\text { Average/below- } \\
\text { average } \\
\text { Risk }\end{array}$ & $\begin{array}{l}\text { Exercise consisted of endurance } \\
\text { training and/or strength training } \\
\text { in supervised classes. Exercise } \\
\text { sessions began with a 10- to } \\
15 \text {-min warm-up and ended } \\
\text { with a 5- to } 10 \text {-min cool-down. } \\
\text { Endurance training used } \\
\text { stationary cycles. } \\
\text { Strength training groups did } \\
\text { resistance exercise of the upper } \\
\text { and lower body using weight } \\
\text { machines. } \\
\text { Subjects received a discharge } \\
\text { planning intervention to } \\
\text { promote continued exercise. } \\
\text { Instructor: NR }\end{array}$ & $\begin{array}{l}\text { Supervised: Strength, } \\
\text { Endurance } \\
\text { - } 1 \mathrm{hr}, 3 \text { times per } \\
\text { week, } 24-26 \text { weeks }\end{array}$ & $\begin{array}{l}\text { Participants } \\
\text { attended } 95 \% \\
\text { of scheduled } \\
\text { exercise sessions }\end{array}$ & $\begin{array}{l}3 \% \text { per } \\
\text { month }\end{array}$ & $\begin{array}{l}\$ 8 \text { per } \\
\text { week }\end{array}$ \\
\hline
\end{tabular}


Table 2 Summary of exercise programs for included studies (Continued)

\begin{tabular}{|c|c|c|c|c|c|c|c|c|}
\hline $\begin{array}{l}\text { cAuthors (year) } \\
\text { Successful/ } \\
\text { Unsuccessful } \\
\text { Quality Score } \\
\text { (of 10) }^{\mathrm{b}}\end{array}$ & $\begin{array}{l}\text { Exercise setting; } \\
\text { Standardized (S), } \\
\text { Individualized (I), } \\
\text { Semi- } \\
\text { individualized (SI) }\end{array}$ & $\begin{array}{l}\text { Population } \\
\text { and } \\
\text { recruitment }\end{array}$ & $\begin{array}{l}\text { Above-average } \\
\text { risk or Average/ } \\
\text { below-average } \\
\text { risk }^{c}\end{array}$ & $\begin{array}{l}\text { Exercise description } \\
\text { and Instructor }\end{array}$ & $\begin{array}{l}\text { Supervised/ } \\
\text { Unsupervised: Exercise } \\
\text { components }^{d} \\
\text { Time, Frequency and } \\
\text { Program Duration }\end{array}$ & Adherence & $\begin{array}{l}\text { Attrition } \\
\text { rate }\end{array}$ & $\begin{array}{l}\text { Exercise } \\
\text { instruction } \\
\text { cost per } \\
\text { participant }\end{array}$ \\
\hline $\begin{array}{l}\text { Bunout et al. } \\
\text { (2005) } \\
\text { Unsuccessful } 5\end{array}$ & Class; SI & $\begin{array}{l}\text { Elderly women } \\
\text { and men; } \\
\text { recruitment } \\
\text { not reported }\end{array}$ & $\begin{array}{l}\text { Average/ } \\
\text { below-average } \\
\text { Risk }\end{array}$ & $\begin{array}{l}\text { Moderate-intensity resistance } \\
\text { exercise training program } \\
\text { included functional weight } \\
\text { bearing exercises, squats, } \\
\text { step-ups in stair, and arm } \\
\text { pull-ups. Participants also } \\
\text { engaged in walking before } \\
\text { and after resistance training. } \\
\text { Instructor: specialized coach }\end{array}$ & $\begin{array}{l}\text { Supervised: Strength, } \\
\text { General physical } \\
\text { activity } \\
\cdot 1 \text { hr, } 2 \text { times per } \\
\text { week, } 1 \text { year }\end{array}$ & $\begin{array}{l}\text { Participants } \\
\text { attended } 52 \% \\
\text { of sessions }\end{array}$ & $N R$ & $\begin{array}{l}\$ 3 \text { per } \\
\text { week }\end{array}$ \\
\hline $\begin{array}{l}\text { Campbell et al. } \\
\text { (1997, 1999a) } \\
\text { Successful } \\
\text { (Campbell and } \\
\text { Robertson 2010; } \\
\text { Campbell and } \\
\text { Robertson 2007; } \\
\text { Gardner et al. 2001; } \\
\text { Robertson and } \\
\text { Campbell 2008; } \\
\text { Robertson et al. } \\
\text { 2002) } 8\end{array}$ & Home; I & $\begin{array}{l}\text { Women aged } \\
80 \text { and older } \\
\text { identified from } \\
\text { registers of general } \\
\text { practices; invited } \\
\text { by PCP to participate }\end{array}$ & $\begin{array}{l}\text { Above-average } \\
\text { Risk }\end{array}$ & $\begin{array}{l}\text { Otago: Physiotherapist } \\
\text { prescribed exercises individually } \\
\text { during } 4 \text { visits over first } 2 \text { months. } \\
\text { Program consisted of muscle } \\
\text { strength, balance retraining, and } \\
\text { walking program. Level of difficulty } \\
\text { increased by increasing number } \\
\text { of repetitions and weights. } \\
\text { Instructor: physiotherapist }\end{array}$ & $\begin{array}{l}\text { Supervised: Balance, } \\
\text { Strength } \\
\cdot 1 \text { hr, } 4 \text { times over } \\
1 \text { st } 2 \text { months } \\
\text { Unsupervised: Balance, } \\
\text { Strength } \\
\cdot 30 \text { min, } 3 \text { times per } \\
\text { week, } 1 \text { year } \\
\text { Unsupervised: General } \\
\text { physical activity } \\
\cdot 2 \text { times per week, } \\
1 \text { year }\end{array}$ & $\begin{array}{l}\text { At end, } 44 \% \text { of total } \\
\text { were still exercising } \\
3+\text { times per week }\end{array}$ & $\begin{array}{l}4 \% \text { per } \\
\text { month } \\
\text { during } \\
\text { second } \\
\text { year }\end{array}$ & $\begin{array}{l}\$ 20 \text { per } \\
\text { week }\end{array}$ \\
\hline $\begin{array}{l}\text { Campbell et al. } \\
\text { (1999b) } \\
\text { Successful } \\
\text { (Campbell and } \\
\text { Robertson 2010; } \\
\text { Campbell and } \\
\text { Robertson 2007; } \\
\text { Gardner et al. } \\
\text { 2001; } \\
\text { Robertson and } \\
\text { Campbell 2008; } \\
\text { Robertson et al. } \\
\text { 2002) } 6\end{array}$ & Home; I & $\begin{array}{l}\text { Women and men } \\
\text { aged } 65 \text { and older } \\
\text { taking antidepressant } \\
\text { or tranquilizer } \\
\text { identified from } \\
\text { register of general } \\
\text { practice groups; } \\
\text { invited by PCP }\end{array}$ & $\begin{array}{l}\text { Above-average } \\
\text { Risk }\end{array}$ & $\begin{array}{l}\text { Otago: Physiotherapist prescribed } \\
\text { exercises individually during } 4 \text { visits } \\
\text { over first } 2 \text { months. Program } \\
\text { consisted of muscle strength, } \\
\text { balance retraining, and walking } \\
\text { program. Level of difficulty } \\
\text { increased by increasing number } \\
\text { of repetitions and weights. } \\
\text { Also included Medication } \\
\text { withdrawal component. } \\
\text { Instructor: physiotherapist }\end{array}$ & $\begin{array}{l}\text { Supervised: Balance, } \\
\text { Strength } \\
\cdot 1 \text { hr, } 4 \text { times over } \\
1 \text { st } 2 \text { months } \\
\text { Unsupervised: Balance, } \\
\text { Strength } \\
\cdot 30 \text { min, } 3 \text { times per } \\
\text { week, } 44 \text { weeks } \\
\text { Unsupervised: General } \\
\text { physical activity } \\
\cdot 2 \text { times per week, } \\
44 \text { weeks }\end{array}$ & $\begin{array}{l}\text { After } 44 \text { weeks, } 63 \% \\
\text { of those remaining } \\
\text { completed their } \\
\text { exercise } 3+\text { times per } \\
\text { week, } 72 \% \text { walked } \\
2+\text { times per week }\end{array}$ & $\begin{array}{l}5 \% \text { per } \\
\text { month }\end{array}$ & $\begin{array}{l}\$ 20 \text { per } \\
\text { week }\end{array}$ \\
\hline $\begin{array}{l}\text { Campbell (2005) } \\
\text { Unsuccessful } \\
\text { (Campbell and } \\
\text { Robertson 2010; } \\
\text { Campbell and } \\
\text { Robertson 2007; } \\
\text { Gardner et al. 2001; } \\
\text { Robertson and } \\
\text { Campbell 2008) } 8\end{array}$ & Home; I & $\begin{array}{l}\text { Women and men } \\
\text { aged } 75 \text { years and } \\
\text { older with poor } \\
\text { vision from register, } \\
\text { optometry clinic, } \\
\text { and low vision } \\
\text { outpatient clinics }\end{array}$ & $\begin{array}{l}\text { Above-average } \\
\text { Risk }\end{array}$ & $\begin{array}{l}\text { Otago: Physiotherapist prescribed } \\
\text { exercises individually during } \\
5 \text { visits over } 6 \text { months. Program } \\
\text { consisted of muscle strength, } \\
\text { balance retraining, and walking } \\
\text { program. Level of difficulty } \\
\text { increased by increasing number } \\
\text { of repetitions and weights. } \\
\text { Program was modified for those } \\
\text { with severe visual acuity loss. } \\
\text { Instructor: physiotherapist }\end{array}$ & $\begin{array}{l}\text { Supervised: Balance, } \\
\text { Strength } \\
\cdot 1 \text { hr, } 5 \text { times over } \\
6 \text { months } \\
\text { Unsupervised: Balance, } \\
\text { Strength } \\
\cdot 30 \text { min, } 3 \text { times per } \\
\text { week, } 1 \text { year } \\
\text { Unsupervised: General } \\
\text { physical activity } \\
\cdot 2 \text { times per week, } \\
1 \text { year }\end{array}$ & $\begin{array}{l}18 \% \text { completed } \\
\text { exercises } 3+\text { times } \\
\text { per week, } 36 \% \\
\text { completed exercises } \\
2 \text { times per week. } \\
44 \% \text { walked } 2+ \\
\text { times per week }\end{array}$ & $\begin{array}{l}1 \% \text { per } \\
\text { month }\end{array}$ & $\begin{array}{l}\$ 8 \text { per } \\
\text { week }\end{array}$ \\
\hline
\end{tabular}


Table 2 Summary of exercise programs for included studies (Continued)

\begin{tabular}{|c|c|c|c|c|c|c|c|c|}
\hline $\begin{array}{l}\text { cAuthors (year) } \\
\text { Successful/ } \\
\text { Unsuccessful }^{a} \\
\text { Quality Score } \\
\text { (of 10) }\end{array}$ & $\begin{array}{l}\text { Exercise setting; } \\
\text { Standardized (S), } \\
\text { Individualized (I), } \\
\text { Semi- } \\
\text { individualized (SI) }\end{array}$ & $\begin{array}{l}\text { Population } \\
\text { and } \\
\text { recruitment }\end{array}$ & $\begin{array}{l}\text { Above-average } \\
\text { risk or Average/ } \\
\text { below-average } \\
\text { risk }^{c}\end{array}$ & $\begin{array}{l}\text { Exercise description } \\
\text { and Instructor }\end{array}$ & $\begin{array}{l}\text { Supervised/ } \\
\text { Unsupervised: Exercise } \\
\text { components } \\
\text { Time, Frequency and } \\
\text { Program Duration }\end{array}$ & Adherence & $\begin{array}{l}\text { Attrition } \\
\text { rate }\end{array}$ & $\begin{array}{l}\text { Exercise } \\
\text { instruction } \\
\text { cost per } \\
\text { participant }\end{array}$ \\
\hline $\begin{array}{l}\text { Carter (2002) } \\
\text { Successful } \\
\text { (Campbell 2002; } \\
\text { Carter et al. 2001) } 5\end{array}$ & Class; S & $\begin{array}{l}\text { Women with } \\
\text { osteoporosis recruited } \\
\text { from those diagnosed } \\
\text { at health center }\end{array}$ & $\begin{array}{l}\text { Above-average } \\
\text { Risk }\end{array}$ & $\begin{array}{l}\text { Osteofit targets posture, balance, } \\
\text { gait, coordination, and hip } \\
\text { and trunk stabilization with } \\
8 \text { to } 16 \text { strengthening and } \\
\text { stretching exercises and strength } \\
\text { training. } \\
\text { Instructor: certified by hospital } \\
\text { osteoporosis program }\end{array}$ & $\begin{array}{l}\text { Supervised: Balance, } \\
\text { Strength, Flexibility } \\
\text { - } 40 \text { min, } 2 \text { times per } \\
\text { week, } 20 \text { weeks }\end{array}$ & $\mathrm{NR}$ & $\begin{array}{l}4 \% \text { per } \\
\text { month }\end{array}$ & $\begin{array}{l}\$ 5 \text { per } \\
\text { week }\end{array}$ \\
\hline $\begin{array}{l}\text { Clemson et al. } \\
\text { (2012) } \\
\text { Successful } \\
\text { (Clemson et al. } \\
\text { 2010) } 7\end{array}$ & Home; I & $\begin{array}{l}\text { Women and men } \\
\text { aged } 70 \text { years or } \\
\text { older with two or } \\
\text { more falls or one } \\
\text { injurious fall in past } \\
12 \text { months; recruited } \\
\text { from Veteran's Affairs } \\
\text { and general practice } \\
\text { databases }\end{array}$ & $\begin{array}{l}\text { Above-average } \\
\text { Risk }\end{array}$ & $\begin{array}{l}\text { Lifestyle integrated Functional } \\
\text { Exercise (LiFE) program included } \\
\text { movements prescribed to } \\
\text { improve balance or increase } \\
\text { strength that are embedded } \\
\text { within everyday activities so } \\
\text { that movements can be done } \\
\text { multiple times per day. } \\
\text { Instructor: physiotherapist }\end{array}$ & $\begin{array}{l}\text { Supervised: Balance, } \\
\text { Strength } \\
\cdot 7 \text { times over } \\
6 \text { months } \\
\text { Unsupervised: Balance, } \\
\text { Strength } \\
\text { - Multiple times per } \\
\text { day, } 7 \text { days per week, } \\
1 \text { year }\end{array}$ & $\begin{array}{l}64 \% \text { completed } \\
\text { exercises at } \\
12 \text { months; } 3.89 \\
\text { mean days per week } \\
\text { exercised in final } \\
\text { month }\end{array}$ & $\begin{array}{l}2 \% \text { per } \\
\text { month }\end{array}$ & $\begin{array}{l}\$ 6 \text { per } \\
\text { week }\end{array}$ \\
\hline $\begin{array}{l}\text { Dangour et al. } \\
\text { (2011) } \\
\text { Unsuccessful } \\
\text { (Dangour et al. } \\
\text { 2007; Walker et al. } \\
\text { 2009) } 7\end{array}$ & Class; SI & $\begin{array}{l}\text { Women and men } \\
\text { aged } 65 \text { to } 67.9 \\
\text { recruited from } \\
\text { health center } \\
\text { catchment areas }\end{array}$ & $\begin{array}{l}\text { Average/below- } \\
\text { average Risk }\end{array}$ & $\begin{array}{l}\text { Physical activity group training } \\
\text { sessions focused on resistance } \\
\text { exercises. Participants were } \\
\text { encouraged to walk to sessions. } \\
\text { Instructor: physical activity } \\
\text { instructor }\end{array}$ & $\begin{array}{l}\text { Supervised: Strength, } \\
\text { General physical } \\
\text { activity } \\
\text { - } 1 \text { hr, } 2 \text { times per } \\
\text { week, } 2 \text { years }\end{array}$ & $\begin{array}{l}38 \% \text { attended at } \\
\text { least } 24 \text { classes over } \\
12 \text { months }\end{array}$ & $\begin{array}{l}1 \% \text { per } \\
\text { month }\end{array}$ & $\begin{array}{l}\text { \$3 per } \\
\text { week }\end{array}$ \\
\hline $\begin{array}{l}\text { Ebrahim et al. (1997) } \\
\text { Unsuccessful } 6\end{array}$ & Home; SI & $\begin{array}{l}\text { Women who had } \\
\text { sustained an upper } \\
\text { arm fracture in past } \\
2 \text { years from registers } \\
\text { of Emergency } \\
\text { Department and } \\
\text { orthopedic clinics of } \\
\text { hospitals; recruited } \\
\text { through letters } \\
\text { explaining study }\end{array}$ & $\begin{array}{l}\text { Above-average } \\
\text { Risk }\end{array}$ & $\begin{array}{l}\text { Participants encouraged to } \\
\text { gradually work up to walking } \\
\text { for } 40 \text { min, } 3 \text { times a week. } \\
\text { Instructed to progressively } \\
\text { increase the amount and speed } \\
\text { of walking. Seen every } 3 \text { months } \\
\text { to discuss problems, reinforce } \\
\text { intervention and allow } \\
\text { physiological measurements to } \\
\text { be taken. } \\
\text { Instructor: nurse }\end{array}$ & $\begin{array}{l}\text { Unsupervised: } \\
\text { Endurance } \\
\text { - } 40 \text { min, } 3 \text { times per } \\
\text { week, } 2 \text { years }\end{array}$ & $\begin{array}{l}\text { All who remained in } \\
\text { trial reported regular } \\
\text { walking }\end{array}$ & $\begin{array}{l}3 \% \text { per } \\
\text { month } \\
\text { during } \\
\text { year } 1\end{array}$ & $\begin{array}{l}\text { \$3 per } \\
\text { week }\end{array}$ \\
\hline
\end{tabular}


Table 2 Summary of exercise programs for included studies (Continued)

\begin{tabular}{|c|c|c|c|c|c|c|c|c|}
\hline $\begin{array}{l}\text { cAuthors (year) } \\
\text { Successful/ } \\
\text { Unsuccessful }^{a} \\
\text { Quality Score } \\
\text { (of 10) }\end{array}$ & $\begin{array}{l}\text { Exercise setting; } \\
\text { Standardized (S), } \\
\text { Individualized (I), } \\
\text { Semi- } \\
\text { individualized (SI) }\end{array}$ & $\begin{array}{l}\text { Population } \\
\text { and } \\
\text { recruitment }\end{array}$ & $\begin{array}{l}\text { Above-average } \\
\text { risk or Average/ } \\
\text { below-average } \\
\text { risk }^{c}\end{array}$ & $\begin{array}{l}\text { Exercise description } \\
\text { and Instructor }\end{array}$ & $\begin{array}{l}\text { Supervised/ } \\
\text { Unsupervised: Exercise } \\
\text { components } \\
\text { Time, Frequency and } \\
\text { Program Duration }\end{array}$ & Adherence & $\begin{array}{l}\text { Attrition } \\
\text { rate }\end{array}$ & $\begin{array}{l}\text { Exercise } \\
\text { instruction } \\
\text { cost per } \\
\text { participant }\end{array}$ \\
\hline $\begin{array}{l}\text { Freiberger et al. } \\
\text { (2012) } \\
\text { Successful (Fitness } \\
\text { group) (Freiberger } \\
\text { et al. 2007) } 8\end{array}$ & $\begin{array}{l}\text { Class and } \\
\text { Home; SI }\end{array}$ & $\begin{array}{l}\text { Women and men } \\
\text { aged } 70+\text { who had } \\
\text { fallen in past } \\
6 \text { months or fear of } \\
\text { falling from health } \\
\text { insurance company } \\
\text { membership } \\
\text { database; recruited } \\
\text { through } \\
\text { questionnaires }\end{array}$ & $\begin{array}{l}\text { Above-average } \\
\text { Risk }\end{array}$ & $\begin{array}{l}\text { All } 3 \text { interventions included } \\
\text { strength and balance exercises } \\
\text { but differed regarding their } \\
\text { second feature (Additional } \\
\text { strength and balance; endurance; } \\
\text { fall risk education). Interventions } \\
\text { were progressive over time and } \\
\text { included intro discussion, warm-up } \\
\text { exercise, main program, cool-down, } \\
\text { and discussion. For home, participants } \\
\text { received brochure describing how } \\
\text { to perform the strength, balance, and } \\
\text { gait exercises. } \\
\text { Instructor: Fall prevention instructors }\end{array}$ & $\begin{array}{l}\text { Strength and balance } \\
\text { group: } \\
\text { Supervised: Balance, } \\
\text { Strength } \\
\text { • } 1 \text { hr, } 2 \text { times per } \\
\text { week, } 4 \text { months } \\
\text { Unsupervised: Balance, } \\
\text { Strength } \\
\text { - Daily } \\
\text { Fitness group: } \\
\text { Supervised: Balance, } \\
\text { Strength, Endurance } \\
\text { - } 1 \text { hr, } 2 \text { times per } \\
\text { week, } 4 \text { months } \\
\text { Unsupervised: Balance, } \\
\text { Strength } \\
\text { - Daily } \\
\text { Multifaceted group: } \\
\text { Supervised: Balance, } \\
\text { Strength } \\
\text { - } 1 \text { hr, } 2 \text { times per } \\
\text { week, } 4 \text { months } \\
\text { Unsupervised: Balance, } \\
\text { Strength } \\
\text { • Daily }\end{array}$ & $\begin{array}{l}82 \% \text { of strength and } \\
\text { balance group, } 84 \% \\
\text { of fitness group, and } \\
84 \% \text { of multifaceted } \\
\text { group attended at } \\
\text { least } 24 \text { of the } 32 \\
\text { sessions }\end{array}$ & $\begin{array}{l}1 \% \text { per } \\
\text { month }\end{array}$ & $\begin{array}{l}\$ 6 \text { per } \\
\text { week }\end{array}$ \\
\hline $\begin{array}{l}\text { Green et al. (2002) } \\
\text { Unsuccessful } \\
\text { (De Weerdt and } \\
\text { Feys 2002; } \\
\text { Dowswell et al. } \\
\text { 2002; Green et al. } \\
\text { 2004) } 8\end{array}$ & $\begin{array}{l}\text { Outpatient rehab } \\
\text { center or Home; I }\end{array}$ & $\begin{array}{l}\text { Women and men } \\
\text { who had a stroke } \\
\text { at least } 1 \text { year } \\
\text { previously and an } \\
\text { associated persisting } \\
\text { mobility problem; } \\
\text { recruited from } \\
\text { hospital and } \\
\text { community therapy } \\
\text { stroke registers }\end{array}$ & $\begin{array}{l}\text { Above-average } \\
\text { Risk }\end{array}$ & $\begin{array}{l}\text { Patients were assessed by a } \\
\text { physiotherapist and then treated } \\
\text { with a problem solving approach } \\
\text { at home or in outpatient } \\
\text { rehabilitation centers. The main } \\
\text { interventions given were gait } \\
\text { re-education, } \\
\text { exercise therapy, functional } \\
\text { exercises, and balance } \\
\text { re-education } \\
\text { Instructor: physiotherapist }\end{array}$ & $\begin{array}{l}\text { Supervised: Balance } \\
\text { - At least } 3 \text { contacts } \\
\text { over } 13 \text { week period }\end{array}$ & $\begin{array}{l}\text { Median: } 3 \text { treatments } \\
\text { per patient }\end{array}$ & $\begin{array}{l}2 \% \text { per } \\
\text { month }\end{array}$ & $\begin{array}{l}\$ 7 \text { per } \\
\text { week }\end{array}$ \\
\hline $\begin{array}{l}\text { Haines et al. } \\
\text { (2009) } \\
\text { Successful } 7\end{array}$ & Home; SI & $\begin{array}{l}\text { Women and men } \\
\text { treated on geriatric } \\
\text { rehabilitation, } \\
\text { medical, or surgical } \\
\text { units of hospital } \\
\text { with gait instability; } \\
\text { physiotherapist } \\
\text { identified patients } \\
\text { and referred to } \\
\text { research team }\end{array}$ & $\begin{array}{l}\text { Above-average } \\
\text { Risk }\end{array}$ & $\begin{array}{l}\text { First home visit provided } \\
\text { instruction for the Kitchen } \\
\text { Table Exercise Program. } \\
\text { Program consisted of DVD } \\
\text { and workbook for progressive } \\
\text { exercise program combining } \\
\text { lower limb strength and balance } \\
\text { exercises. Program included } \\
6 \text { types of exercises each with } \\
6 \text { different levels of difficulties. } \\
\text { Instructor: physiotherapist. }\end{array}$ & $\begin{array}{l}\text { Supervised: Balance, } \\
\text { Strength } \\
\text { - At least initial and } \\
\text { follow up visit } \\
\text { Unsupervised: Balance, } \\
\text { Strength } \\
\text { - } 2 \text { times per week, } \\
8 \text { weeks }\end{array}$ & $\begin{array}{l}15 \text { of } 19 \text { attempted } \\
\text { the program at least } \\
\text { once during week } 1 ; \\
8 \text { attempted } 1+ \\
\text { times during week } 8\end{array}$ & $\begin{array}{l}0 \% \text { per } \\
\text { month }\end{array}$ & $\begin{array}{l}\$ 10 \text { per } \\
\text { week }\end{array}$ \\
\hline
\end{tabular}


Table 2 Summary of exercise programs for included studies (Continued)

\begin{tabular}{|c|c|c|c|c|c|c|c|c|}
\hline $\begin{array}{l}\text { CAuthors (year) } \\
\text { Successful// } \\
\text { Unsuccessful }^{a} \\
\text { Quality Score } \\
\text { (of 10) }\end{array}$ & $\begin{array}{l}\text { Exercise setting; } \\
\text { Standardized (S), } \\
\text { Individualized (I), } \\
\text { Semi- } \\
\text { individualized (SI) }\end{array}$ & $\begin{array}{l}\text { Population } \\
\text { and } \\
\text { recruitment }\end{array}$ & $\begin{array}{l}\text { Above-average } \\
\text { risk or Average/ } \\
\text { below-average } \\
\text { risk }^{c}\end{array}$ & $\begin{array}{l}\text { Exercise description } \\
\text { and Instructor }\end{array}$ & $\begin{array}{l}\text { Supervised/ } \\
\text { Unsupervised: Exercise } \\
\text { components }^{d} \\
\text { Time, Frequency and } \\
\text { Program Duration }\end{array}$ & Adherence & $\begin{array}{l}\text { Attrition } \\
\text { rate }\end{array}$ & $\begin{array}{l}\text { Exercise } \\
\text { instruction } \\
\text { cost per } \\
\text { participant }\end{array}$ \\
\hline $\begin{array}{l}\text { Hauer et al. (2001) } \\
\text { Successful } 6\end{array}$ & $\begin{array}{l}\text { Class at outpatient } \\
\text { rehab unit; SI }\end{array}$ & $\begin{array}{l}\text { Women with fall as } \\
\text { reason for admission } \\
\text { to hospital or recent } \\
\text { history of injurious } \\
\text { fall; recruited at end } \\
\text { of rehabilitation } \\
\text { from hospital }\end{array}$ & $\begin{array}{l}\text { Above-average } \\
\text { Risk }\end{array}$ & $\begin{array}{l}\text { Ambulatory training of strength, } \\
\text { functional performance, and } \\
\text { balance. Exercise included warm } \\
\text { up on stationary cycles, high-intensity } \\
\text { progressive resistance training of } \\
\text { functionally relevant muscle groups, } \\
\text { and training in walking, stepping, } \\
\text { and sitting to modify unsafe or } \\
\text { inefficient performance. Patients } \\
\text { progressed to advanced levels } \\
\text { of exercise. Physiotherapy included } \\
\text { massaging, stretching, and heat/ice } \\
\text { to orthopedic problems. } \\
\text { Instructor: therapeutic recreation } \\
\text { specialist. }\end{array}$ & $\begin{array}{l}\text { Supervised: Balance, } \\
\text { Strength } \\
\cdot 2.25 \text { hr, } 3 \text { times per } \\
\text { week, } 3 \text { months } \\
\text { Supervised: Flexibility, } \\
\text { Other } \\
\cdot 25 \text { min sessions, } 2 \\
\text { times per week, } \\
3 \text { months }\end{array}$ & $85 \%$ adherence & $\begin{array}{l}8 \% \text { per } \\
\text { month }\end{array}$ & $\begin{array}{l}\$ 50 \text { per } \\
\text { week }\end{array}$ \\
\hline $\begin{array}{l}\text { Hornbrook et al. } \\
\text { (1994) } \\
\text { Successful } \\
\text { (Stevens et al. } \\
\text { 1991; Stevens } \\
\text { et al. 1992) } 5\end{array}$ & $\begin{array}{l}\text { Class and } \\
\text { Home; S }\end{array}$ & $\begin{array}{l}\text { Members of large } \\
\text { HMO aged } 65 \text { years } \\
\text { and older; Letter } \\
\text { sent with follow up } \\
\text { phone call or home } \\
\text { visit if no response }\end{array}$ & $\begin{array}{l}\text { Average/below- } \\
\text { average Risk }\end{array}$ & $\begin{array}{l}\text { Weekly group meetings included } \\
\text { didactic presentations, demonstrations } \\
\text { of falls prevention exercises, and small } \\
\text { group meetings. Exercises were chosen } \\
\text { to provide active involvement of all } \\
\text { body parts, maintain full range of } \\
\text { motion of all joints, provide } \\
\text { strengthening, improve posture by } \\
\text { preventing forward flexion of the head } \\
\text { and shoulders, and improve balance. } \\
\text { Participants were given a manual } \\
\text { describing the exercises to follow at } \\
\text { home and encouraged } \\
\text { to begin walking. After the first } 4 \text { sessions, } \\
\text { quarterly maintenance sessions were held. } \\
\text { Instructor: health behaviorist and } \\
\text { physical therapist }\end{array}$ & $\begin{array}{l}\text { Supervised: Balance, } \\
\text { Strength } \\
\text { - } 90 \text { min, } 1 \text { time per } \\
\text { week, } 4 \text { weeks } \\
\text { Unsupervised: Balance, } \\
\text { Strength } \\
\text { - Time not specified } \\
\text { Unsupervised: General } \\
\text { physical activity } \\
\text { - } 3 \text { times per week }\end{array}$ & $\begin{array}{l}78 \% \text { attended at } \\
\text { least } 1 \text { session, } 61 \% \\
\text { attended } 3+\text { sessions }\end{array}$ & NR & $\begin{array}{l}\text { \$4 per } \\
\text { week }\end{array}$ \\
\hline $\begin{array}{l}\text { Iwamoto et al. } \\
\text { (2009) } \\
\text { Successful } 6\end{array}$ & $\begin{array}{l}\text { Clinic or } \\
\text { hospital; S }\end{array}$ & $\begin{array}{l}\text { Women and men } \\
\text { aged } 50 \text { or older } \\
\text { who visited hospital } \\
\text { Department of } \\
\text { Orthopedic Surgery } \\
\text { or clinic }\end{array}$ & $\begin{array}{l}\text { Above-average } \\
\text { Risk }\end{array}$ & $\begin{array}{l}\text { Exercise program in clinic or hospital } \\
\text { consisted of calisthenics, body balance } \\
\text { training, muscle power training, and } \\
\text { walking ability training. } \\
\text { Instructor: NR }\end{array}$ & $\begin{array}{l}\text { Supervised: Balance, } \\
\text { Strength, Flexibility } \\
\cdot 30 \text { min, } 3 \text { times per } \\
\text { week, } 5 \text { months }\end{array}$ & $\begin{array}{l}\text { Compliance with } \\
\text { exercise } 100 \%\end{array}$ & $0 \%$ & $\begin{array}{l}\$ 62 \text { per } \\
\text { week }\end{array}$ \\
\hline
\end{tabular}

or Clinic erformance, and

walking, stepping,

o orthopedic problems.

specialist.

Average/below- Weekly group meetings included group meetins. Ex

parts, maintain full range of strengthening improve posture by preventing forward flexion of the head

to begin walking. After the first 4 sessions, quarterly maintenance sessions were held. instructor: health behaviorist and walking ability training. 30 min, 3 times per week, 5 months 
Table 2 Summary of exercise programs for included studies (Continued)

\begin{tabular}{|c|c|c|c|c|c|c|c|c|}
\hline $\begin{array}{l}\text { CAuthors (year) } \\
\text { Successful/ } \\
\text { Unsuccessful } \\
\text { Quality Score } \\
\text { (of 10) }\end{array}$ & $\begin{array}{l}\text { Exercise setting; } \\
\text { Standardized (S), } \\
\text { Individualized (I), } \\
\text { Semi- } \\
\text { individualized (SI) }\end{array}$ & $\begin{array}{l}\text { Population } \\
\text { and } \\
\text { recruitment }\end{array}$ & $\begin{array}{l}\text { Above-average } \\
\text { risk or Average/ } \\
\text { below-average } \\
\text { risk }\end{array}$ & $\begin{array}{l}\text { Exercise description } \\
\text { and Instructor }\end{array}$ & $\begin{array}{l}\text { Supervised/ } \\
\text { Unsupervised: Exercise } \\
\text { components }^{\mathrm{d}} \\
\text { Time, Frequency and } \\
\text { Program Duration }\end{array}$ & Adherence & $\begin{array}{l}\text { Attrition } \\
\text { rate }\end{array}$ & $\begin{array}{l}\text { Exercise } \\
\text { instruction } \\
\text { cost per } \\
\text { participant }\end{array}$ \\
\hline $\begin{array}{l}\text { Kronhed et al. } \\
\text { (2009) } \\
\text { Successful } \\
\text { (Kronhed 2009) } 7\end{array}$ & Class; I & $\begin{array}{l}\text { Women with } \\
\text { established } \\
\text { osteoporosis and at } \\
\text { least one fragility } \\
\text { fracture identified } \\
\text { from files at the } \\
\text { Osteoporosis Unit } \\
\text { at hospital; invitation } \\
\text { letter sent }\end{array}$ & $\begin{array}{l}\text { Average/below- } \\
\text { average Risk }\end{array}$ & $\begin{array}{l}\text { Exercise consisted of a strength } \\
\text { training program supervised by } \\
\text { a physiotherapist. The program } \\
\text { consisted of a warm-up using } \\
\text { exercise bicycles and a cross-trainer. } \\
\text { Back strengthening exercises, } \\
\text { abdominal muscle training, sequence } \\
\text { training exercises, and } \\
\text { balance exercises were performed. } \\
\text { In the introductory instruction, } \\
\text { participants received personal } \\
\text { instruction and an individually } \\
\text { designed load that was progressively } \\
\text { increased according to the } \\
\text { participant's capacity. Sessions finished } \\
\text { with } 10 \text { min of stretching. Participants } \\
\text { were encouraged to continue the } \\
\text { training exercise program on their } \\
\text { own at senior gyms after the } \\
\text { supervised group exercise training } \\
\text { period. } \\
\text { Instructor: physiotherapist }\end{array}$ & $\begin{array}{l}\text { Supervised: Balance, } \\
\text { Strength, Flexibility, } \\
\text { Endurance } \\
\cdot 1 \text { hr, } 2 \text { times per } \\
\text { week, } 4 \text { months }\end{array}$ & $\begin{array}{l}\text { Participants } \\
\text { completed an } \\
\text { average of } 24 \text { of } 30 \\
\text { sessions }\end{array}$ & $\begin{array}{l}5 \% \text { per } \\
\text { month }\end{array}$ & $\begin{array}{l}\text { \$3 per } \\
\text { week }\end{array}$ \\
\hline $\begin{array}{l}\text { Latham et al. } \\
\text { (2003a) } \\
\text { Unsuccessful } \\
\text { (Judge 2003; } \\
\text { Latham 2003b) } 8\end{array}$ & $\begin{array}{l}\text { Hospital and } \\
\text { Home; SI }\end{array}$ & $\begin{array}{l}\text { Frail women and } \\
\text { men aged } 65 \text { and } \\
\text { older admitted to } \\
\text { geriatric rehabilitation } \\
\text { units }\end{array}$ & $\begin{array}{l}\text { Above-average } \\
\text { Risk }\end{array}$ & $\begin{array}{l}\text { Resistance exercise consisted of a } \\
\text { quadriceps exercise program using } \\
\text { adjustable ankle cuff weights. } \\
\text { The aim was for patients to exercise } \\
\text { at a high intensity by midway through } \\
\text { the program. Each session began with } \\
\text { individualized warm-up stretches, } \\
\text { followed knee extensions. Most of the } \\
\text { patients performed their first two exercise } \\
\text { sessions in the hospital and continued } \\
\text { the rest of their sessions at home. } \\
\text { Instructor: physical therapist }\end{array}$ & $\begin{array}{l}\text { Supervised: Strength } \\
\cdot 2 \text { sessions, then } \\
\text { biweekly } \\
\text { Unsupervised: Strength } \\
\cdot 3 \text { times per week, } \\
10 \text { weeks }\end{array}$ & $\begin{array}{l}\text { Patients adhered to } \\
82 \% \text { of prescribed } \\
\text { sessions }\end{array}$ & $\begin{array}{l}3 \% \text { per } \\
\text { month }\end{array}$ & $\begin{array}{l}\$ 29 \text { per } \\
\text { week }\end{array}$ \\
\hline $\begin{array}{l}\text { Li et al. (2005) } \\
\text { Successful } \\
\text { (Harmer and Li } \\
\text { 2008; Li et al. } \\
\text { 2004; Li et al. } \\
\text { 2008) } 5\end{array}$ & Class; S & $\begin{array}{l}\text { Women and men } \\
\text { aged } 70 \text { years or } \\
\text { older enrolled in } \\
\text { non-profit hospital } \\
\text { system; recruited } \\
\text { through letter sent } \\
\text { by PCP, follow-up } \\
\text { call from research staff. }\end{array}$ & $\begin{array}{l}\text { Above-average } \\
\text { Risk }\end{array}$ & $\begin{array}{l}\text { Classical Yang Style Tai Chi } \\
(24 \text { forms) classes emphasize } \\
\text { multidirectional weight shifting, } \\
\text { awareness of body alignment, and } \\
\text { movement coordination. } \\
\text { Instructor: experienced Tai Chi instructor }\end{array}$ & $\begin{array}{l}\text { Supervised: 3D } \\
\cdot 1 \text { hr, } 3 \text { times per } \\
\text { week, } 26 \text { weeks }\end{array}$ & $\begin{array}{l}\text { Median compliance } \\
\text { was } 61 \text { of } 78 \text { sessions }\end{array}$ & $\begin{array}{l}5 \% \text { per } \\
\text { month }\end{array}$ & $\begin{array}{l}\$ 4 \text { per } \\
\text { week }\end{array}$ \\
\hline
\end{tabular}


Table 2 Summary of exercise programs for included studies (Continued)

\begin{tabular}{|c|c|c|c|c|c|c|c|c|}
\hline $\begin{array}{l}\text { CAuthors (year) } \\
\text { Successful/ } \\
\text { Unsuccessful } \\
\text { Quality Score } \\
\text { (of 10) }\end{array}$ & $\begin{array}{l}\text { Exercise setting; } \\
\text { Standardized (S), } \\
\text { Individualized (I), } \\
\text { Semi- } \\
\text { individualized (SI) }\end{array}$ & $\begin{array}{l}\text { Population } \\
\text { and } \\
\text { recruitment }\end{array}$ & $\begin{array}{l}\text { Above-average } \\
\text { risk or Average/ } \\
\text { below-average } \\
\text { risk }\end{array}$ & $\begin{array}{l}\text { Exercise description } \\
\text { and Instructor }\end{array}$ & $\begin{array}{l}\text { Supervised/ } \\
\text { Unsupervised: Exercise } \\
\text { components }^{d} \\
\text { Time, Frequency and } \\
\text { Program Duration }\end{array}$ & Adherence & $\begin{array}{l}\text { Attrition } \\
\text { rate }\end{array}$ & $\begin{array}{l}\text { Exercise } \\
\text { instruction } \\
\text { cost per } \\
\text { participant }^{\mathrm{e}}\end{array}$ \\
\hline $\begin{array}{l}\text { Lin et al. (2007) } \\
\text { Successful } 5\end{array}$ & Home; I & $\begin{array}{l}\text { Women and men } \\
\text { aged } 65 \text { and older } \\
\text { who had medical } \\
\text { attention due to a } \\
\text { fall; recruited from } \\
\text { clinics and hospitals }\end{array}$ & $\begin{array}{l}\text { Average/below- } \\
\text { average Risk }\end{array}$ & $\begin{array}{l}\text { Exercise consisted of stretching, muscle } \\
\text { strengthening, and balance training at } \\
\text { increasing levels of difficulty. The training } \\
\text { was individualized for each participant } \\
\text { and consisted of } 10 \text { min of warm-up, } \\
30 \text { min of exercise, and } 10 \text { min } \\
\text { of cool-down. } \\
\text { Participants were instructed to } \\
\text { practice these exercises at least three } \\
\text { times a week. } \\
\text { Instructor: physical therapist }\end{array}$ & $\begin{array}{l}\text { Supervised: Balance, } \\
\text { Strength, Flexibility } \\
\text { • Every } 2 \text { weeks, } \\
4 \text { months } \\
\text { Unsupervised: Balance, } \\
\text { Strength, Flexibility } \\
\text { - } 40-60 \text { min, } 3 \text { times } \\
\text { per week, } 4 \text { months }\end{array}$ & $N R$ & $\begin{array}{l}6 \% \text { per } \\
\text { month }\end{array}$ & $\begin{array}{l}\text { \$17 per } \\
\text { week }\end{array}$ \\
\hline $\begin{array}{l}\text { Logghe et al. } \\
\text { (2009) } \\
\text { Unsuccessful } \\
\text { (Logghe et al. } \\
\text { 2011; Zeeuwe } \\
\text { et al. 2006) } 8\end{array}$ & $\begin{array}{l}\text { Class and } \\
\text { Home; S }\end{array}$ & $\begin{array}{l}\text { Women and men } \\
\text { aged } 70 \text { years and } \\
\text { older; recruited from } \\
\text { patient registration } \\
\text { files of PCPs; letters } \\
\text { sent by PCP }\end{array}$ & $\begin{array}{l}\text { Above-average } \\
\text { Risk }\end{array}$ & $\begin{array}{l}\text { Tai Chi Chuan training with ten positions. } \\
\text { Participants asked to practice the Tai Chi } \\
\text { Chuan positions at home. } \\
\text { Instructor: Tai Chi Chuan instructor }\end{array}$ & $\begin{array}{l}\text { Supervised: 3D } \\
\cdot 1 \text { hr, } 2 \text { times per } \\
\text { week, } 13 \text { weeks } \\
\text { Unsupervised: } 3 \mathrm{D} \\
\cdot 15 \text { min, } 2 \text { times per } \\
\text { week, } 13 \text { weeks }\end{array}$ & $\begin{array}{l}47 \% \text { attended at } \\
\text { least } 80 \% \text { of lessons, } \\
85 \% \text { completed } \\
\text { home exercise }\end{array}$ & $\begin{array}{l}9 \% \text { per } \\
\text { month }\end{array}$ & $\begin{array}{l}\$ 4 \text { per } \\
\text { week }\end{array}$ \\
\hline $\begin{array}{l}\text { Luukinen et a. } \\
\text { (2007) } \\
\text { Unsuccessful } \\
\text { (Lehtola et al. } \\
\text { 2006; Luukinen } \\
\text { et al. 2006) } 6\end{array}$ & $\begin{array}{l}\text { Class and/or } \\
\text { home; I }\end{array}$ & $\begin{array}{l}\text { Women and men } \\
\text { aged } 85 \text { years or } \\
\text { older; Recruitment } \\
\text { not reported }\end{array}$ & $\begin{array}{l}\text { Above-average } \\
\text { Risk }\end{array}$ & $\begin{array}{l}\text { Individual intervention plans were } \\
\text { made during home visits by } \\
\text { physiotherapist and occupational } \\
\text { therapist based on risk factors. } \\
\text { Home and group exercise, and } \\
\text { walking exercises were } \\
\text { recommended. } \\
\text { Instructor: physiotherapist and } \\
\text { occupational therapist }\end{array}$ & $\begin{array}{l}\text { Supervised: General } \\
\text { physical activity } \\
\text { - Time not reported } \\
\text { Unsupervised: not } \\
\text { reported } \\
\cdot 3 \text { times per day, } \\
1.5 \text { years }\end{array}$ & NR & $\begin{array}{l}<1 \% \\
\text { per } \\
\text { month }\end{array}$ & NR \\
\hline $\begin{array}{l}\text { Madureira et al. } \\
\text { (2007) } \\
\text { Successful } 6\end{array}$ & $\begin{array}{l}\text { Class and } \\
\text { Home; S }\end{array}$ & $\begin{array}{l}\text { Women aged } \\
65 \text { years and older; } \\
\text { recruited from } \\
\text { patients of } \\
\text { Osteometabolic } \\
\text { Disease Outpatient } \\
\text { Clinic }\end{array}$ & $\begin{array}{l}\text { Above-average } \\
\text { Risk }\end{array}$ & $\begin{array}{l}\text { Balance Training Program consisted } \\
\text { of warm-up and stretching, walking, } \\
\text { and balance training in dynamic } \\
\text { and static positions. Patients were } \\
\text { encouraged to continue same } \\
\text { exercises at home. } \\
\text { Instructor: experienced physiotherapist }\end{array}$ & $\begin{array}{l}\text { Supervised: Balance, } \\
\text { Flexibility, General } \\
\text { physical activity } \\
\text { - } 1 \text { hr, } 1 \text { time per week, } \\
1 \text { year ( } 40 \text { classes total) } \\
\text { Unsupervised: Balance, } \\
\text { Flexibility, General } \\
\text { physical activity } \\
\text { - } 30 \text { min, } 3 \text { times per } \\
\text { week, } 1 \text { year }\end{array}$ & $\begin{array}{l}60 \% \text { participated in } \\
\text { all classes; } 77 \% \\
\text { completed home } \\
\text { exercises } 1+\text { times } \\
\text { per week }\end{array}$ & $\begin{array}{l}1 \% \text { per } \\
\text { month }\end{array}$ & $\begin{array}{l}\text { \$1 per } \\
\text { week }\end{array}$ \\
\hline $\begin{array}{l}\text { Protas et al. (2005) } \\
\text { Successful } 5\end{array}$ & $\begin{array}{l}\text { Outpatient } \\
\text { research center; I }\end{array}$ & $\begin{array}{l}\text { Men with idiopathic } \\
\text { Parkinson's disease } \\
\text { diagnosed at } \\
\text { Veterans Affairs } \\
\text { center }\end{array}$ & $\begin{array}{l}\text { Above-average } \\
\text { Risk }\end{array}$ & $\begin{array}{l}\text { Gait training consisted of walking on } \\
\text { a treadmill at speed greater than } \\
\text { over ground walking speed while } \\
\text { walking in } 4 \text { directions and supported } \\
\text { in harness for safety. Step training included } \\
\text { suddenly turning treadmill on and off } \\
\text { while subject stood in safety harness. } \\
\text { Instructor: physical therapist }\end{array}$ & $\begin{array}{l}\text { Supervised: Balance } \\
\cdot 1 \mathrm{hr} \text { session, } 3 \text { times } \\
\text { per week, } 8 \text { weeks }\end{array}$ & NR & NR & $\begin{array}{l}\text { \$124 per } \\
\text { week }\end{array}$ \\
\hline
\end{tabular}


Table 2 Summary of exercise programs for included studies (Continued)

\begin{tabular}{|c|c|c|c|c|c|c|c|c|}
\hline $\begin{array}{l}\text { cAuthors (year) } \\
\text { Successful/ } \\
\text { Unsuccessful }^{\mathrm{a}} \\
\text { Quality Score } \\
(\text { of 10) }\end{array}$ & $\begin{array}{l}\text { Exercise setting; } \\
\text { Standardized (S), } \\
\text { Individualized (I), } \\
\text { Semi- } \\
\text { individualized (SI) }\end{array}$ & $\begin{array}{l}\text { Population } \\
\text { and } \\
\text { recruitment }\end{array}$ & $\begin{array}{l}\text { Above-average } \\
\text { risk or Average/ } \\
\text { below-average } \\
\text { risk }^{c}\end{array}$ & $\begin{array}{l}\text { Exercise description } \\
\text { and Instructor }\end{array}$ & $\begin{array}{l}\text { Supervised/ } \\
\text { Unsupervised: Exercise } \\
\text { components }^{d} \\
\text { Time, Frequency and } \\
\text { Program Duration }\end{array}$ & Adherence & $\begin{array}{l}\text { Attrition } \\
\text { rate }\end{array}$ & $\begin{array}{l}\text { Exercise } \\
\text { instruction } \\
\text { cost per } \\
\text { participant }^{\text {e }}\end{array}$ \\
\hline $\begin{array}{l}\text { Robertson et al. } \\
\text { (2001) } \\
\text { Successful } \\
\text { (Campbell and } \\
\text { Robertson 2010; } \\
\text { Campbell and } \\
\text { Robertson 2007; } \\
\text { Gardner et al. } \\
\text { 2001; Robertson } \\
\text { and Campbell } \\
\text { 2008; Robertson } \\
\text { et al. 2002) } 8\end{array}$ & Home; I & $\begin{array}{l}\text { Women and men } \\
\text { aged } 75 \text { and older } \\
\text { from registers at } 17 \\
\text { practices; letter sent } \\
\text { from PCP }\end{array}$ & $\begin{array}{l}\text { Average/below- } \\
\text { average Risk }\end{array}$ & $\begin{array}{l}\text { Otago: Set of muscle strengthening } \\
\text { and balance retraining exercises that } \\
\text { progressed in difficulty and a walking } \\
\text { plan. Program individually prescribed } \\
\text { during } 5 \text { home visits at weeks } 1,3,4 \text {, } \\
\text { and } 8 \text {, and a booster visit after } 6 \text { months. } \\
\text { Instructor: nurse }\end{array}$ & $\begin{array}{l}\text { Supervised: Balance, } \\
\text { Strength } \\
\cdot 5 \text { times } \\
\text { Unsupervised: Balance, } \\
\text { Strength } \\
\cdot 30 \text { min, } 3 \text { times per } \\
\text { week, } 1 \text { year } \\
\text { Unsupervised: General } \\
\text { physical activity } \\
\cdot 1 \text { year }\end{array}$ & $\begin{array}{l}43 \% \text { exercised } 3+ \\
\text { times per week, } 72 \% \\
\text { exercised } 2+\text { times } \\
\text { per week, } 71 \% \\
\text { walked } 2+\text { times per } \\
\text { week }\end{array}$ & $\begin{array}{l}1 \% \text { per } \\
\text { month }\end{array}$ & $\begin{array}{l}\$ 3 \text { per } \\
\text { week }\end{array}$ \\
\hline $\begin{array}{l}\text { Rubenstein et al. } \\
\text { (2000) Successful } \\
7\end{array}$ & Class; S & $\begin{array}{l}\text { Men aged } 70 \text { years } \\
\text { or older with at least } \\
1 \text { key fall risk factor; } \\
\text { recruited through } \\
\text { Veterans Affairs } \\
\text { Ambulatory Care } \\
\text { Center. }\end{array}$ & $\begin{array}{l}\text { Above-average } \\
\text { Risk }\end{array}$ & $\begin{array}{l}\text { Group exercise focused on increasing } \\
\text { strength and endurance and improving } \\
\text { mobility and balance. Strengthening } \\
\text { exercises progressed over first } 4 \text { weeks. } \\
\text { Endurance training included bicycle, } \\
\text { treadmill, and indoor walking. Balance } \\
\text { training increased in difficulty over } \\
12 \text { weeks. } \\
\text { Instructor: exercise physiology graduate } \\
\text { students. }\end{array}$ & $\begin{array}{l}\text { Supervised: Balance, } \\
\text { Strength, Endurance } \\
\text { - } 90 \text { min, } 3 \text { times per } \\
\text { week, } 12 \text { weeks }\end{array}$ & $\begin{array}{l}\text { Participants attended } \\
84 \% \text { of sessions }\end{array}$ & $\begin{array}{l}3 \% \text { per } \\
\text { month }\end{array}$ & $\begin{array}{l}\$ 10 \text { per } \\
\text { week }\end{array}$ \\
\hline $\begin{array}{l}\text { Steadman et al. } \\
\text { (2003) } \\
\text { Unsuccessful } 5\end{array}$ & Hospital; I & $\begin{array}{l}\text { Subjects aged } 60 \\
\text { and older recruited } \\
\text { from attendees at } \\
\text { multidisciplinary falls } \\
\text { clinic }\end{array}$ & $\begin{array}{l}\text { Above-average } \\
\text { Risk }\end{array}$ & $\begin{array}{l}\text { Assisted walking within parallel bars, } \\
\text { assessment for mobility aids, stair } \\
\text { practice, general bed mobility skills, } \\
\text { and transfers. Balance exercises } \\
\text { consisted of repetition } \\
\text { of a series of graded tasks specific } \\
\text { to functional balance with targets } \\
\text { of distance and time to provide } \\
\text { feedback. } \\
\text { Also discussed fall avoidance behaviors } \\
\text { and strategies for coping with a long } \\
\text { time on the floor. } \\
\text { Instructor: clinical physiotherapists }\end{array}$ & $\begin{array}{l}\text { Supervised: Balance } \\
\text { - } 45 \text { min, } 2 \text { times per } \\
\text { week, } 6 \text { weeks }\end{array}$ & $\begin{array}{l}\text { Random selection of } \\
\text { patients showed } \\
\text { protocol being } \\
\text { adhered to }\end{array}$ & $\begin{array}{l}10 \% \\
\text { per } \\
\text { month }\end{array}$ & $\begin{array}{l}\$ 62 \text { per } \\
\text { week }\end{array}$ \\
\hline
\end{tabular}


Table 2 Summary of exercise programs for included studies (Continued)

\begin{tabular}{|c|c|c|c|c|c|c|c|c|}
\hline $\begin{array}{l}\text { Swanenburg et al. } \\
\text { (2007) } \\
\text { Successful } 6\end{array}$ & $\begin{array}{l}\text { Hospital } \\
\text { physiotherapy } \\
\text { dept.; I }\end{array}$ & $\begin{array}{l}\text { Women with } \\
\text { osteoporosis; } \\
\text { recruitment not } \\
\text { reported. }\end{array}$ & $\begin{array}{l}\text { Above-average } \\
\text { Risk }\end{array}$ & $\begin{array}{l}\text { Program tailored to individual during } \\
\text { initial } 2 \text { weeks. Two sessions per week } \\
\text { focused on progressive resistance } \\
\text { training and individual exercises to } \\
\text { improve coordination, balance and } \\
\text { endurance. One session per week } \\
\text { consisted of a group exercise focused } \\
\text { on balance exercises and games. } \\
\text { Participants were also given daily } \\
\text { protein supplements and encouraged } \\
\text { to continue program after initial } 3 \text { months. } \\
\text { Instructor: physical therapist }\end{array}$ & $\begin{array}{l}\text { Supervised: Balance, } \\
\text { Strength, Endurance } \\
\cdot 70 \text { min, } 2 \text { times per } \\
\text { week, } 12 \text { weeks } \\
\text { Supervised: Balance } \\
\cdot 70 \text { min, } 1 \text { time per } \\
\text { week, } 12 \text { weeks }\end{array}$ & $\begin{array}{l}\text { Compliance with } \\
\text { exercise } 93 \%\end{array}$ & $\begin{array}{l}3 \% \text { per } \\
\text { month }\end{array}$ & $\begin{array}{l}\text { \$144 per } \\
\text { week }\end{array}$ \\
\hline
\end{tabular}

$P C P$ primary care provider, NR not reported, HMO Health Maintenance Organization

${ }^{\text {a }}$ Successful/Unsuccessful: Study classified as successful if intervention results were within the pooled estimate of the effect of exercise on the rate of falls in the meta-analysis of exercise interventions (pooled rate ratio $0.84,95 \% \mathrm{Cl} 0.77-0.91)$ by Sherrington et al. 2011

b Quality score: Quality of RCTs was assessed by Physiotherapy Evidence Database (PEDro)

c Above-average risk/Average/below-average risk population: Study sample classified as at above-average risk for falls if baseline annualized probability of at least one fall was higher than $36 \%$ in the sample. This is the upper limit of the $95 \%$ confidence interval for the probability of falling at least once in any given year for an unselected sample of individuals age $\geq 65$ (Ganz et al. 2007)

the upper limit of the $95 \%$ confidence interval for the probability of falling at least once in any given year for an unselected sample
$\mathrm{d}$ Exercise components: Balance: Gait, balance, and functional training; Strength: Strength/resistance; 3D: Tai Chi, Qi Gong, Dance

exercise components: Balance: Gait, balance, and functional training; Strength: Strength/resistance; 3D: Tai Chi, Qi Gong, Dance

reported, median wage of other studies; Physical therapist http://www.bls.gov/oes/current/oes291123.htm; Recreation therapist http://www.bls.gov/oes/current/oes291125.htm; Registered

reported, median wage of other studies; Physical thera
nurse http://www.bls.gov/oes/current/oes291141.htm 
other regular (Campbell et al. 1999b; Campbell 1997) telephone calls once the home visits ceased, or made telephone calls during months with no scheduled home visit (Campbell et al. 2005; Robertson et al. 2001).

\section{Exercise frequency and program duration}

Among successful studies, total weekly exercise time ranged from $80 \mathrm{~min}$ (Carter et al. 2002) to more than $7 \mathrm{~h}$ (Table 2) (Hauer et al. 2001). Most successful studies prescribed exercise three or more times weekly. Authors of one unsuccessful study suggested that "lack of significant improvement in mobility of study participants could have resulted from too few treatments (Green et al. 2002)." As described by one successful program that prescribed 30 min sessions three times a week, programs should consider the population's ability to sustain the intensity of exercise: "the intensity and frequency of the exercise program were considered to be reasonable for the elderly to be continued without any fatigue and difficulty for 5 months (Iwamoto et al. 2009)."

Duration of successful programs ranged from eight weeks to two years. Authors of a successful Tai Chi program noted: "our results indicate that several months of practice were needed before significant decreases in falls occurred, suggesting that no tangible results in falls should be expected from short-term exposure to Tai Chi (i.e., less than 3 months) (Li et al. 2005)." The duration may be particularly important for group exercise programs because "the benefit from the exercises lasts only for as long as the person participates in the program (Campbell 2002)."

\section{Participant adherence}

The level of adherence was highly variable (Table 2). For example, one study reported $18 \%$ of participants completed all prescribed exercises in their home during the second year (Campbell et al. 2005) while another study reported $100 \%$ adherence with supervised exercise sessions at a clinic or hospital for 5 months (Iwamoto et al. 2009).

Barriers to high adherence included unwillingness to change lifestyle and habits (Luukinen et al. 2007) and discomfort (Haines et al. 2009). For group exercises, having a pleasant and convenient location (Logghe et al. 2009; Madureira et al. 2007) and providing transportation or reimbursing travel costs (Hauer et al. 2001; Hornbrook et al. 1994; Logghe et al. 2009) can improve adherence. Authors of a successful class exercise program noted that "A secure environment, session supervision and the opportunity for social interaction reduce the feeling of isolation. A social support system is considered important in group activities, and helps sustain adherence and the effectiveness of the weekly exercise sessions and also the adherence to home-based exercises (Madureira et al. 2007)." The role of transportation, particularly for frail individuals, was described by authors of another successful study: "Relying on participants to provide their own transportation creates a strong selection effect towards persons with higher levels of functioning... Many of the participants aged older than 80 had stopped driving and were not comfortable using public transportation (Stevens et al. 1992)."

Studies also suggested that encouragement and supervision may improve adherence (Bunout et al. 2005; Campbell et al. 1997; Madureira et al. 2007). In classes, instructors can provide direct supervision of participants performing the exercises. Calls to individuals who miss classes can provide "active reinforcement to encourage participation in the program (Bunout et al. 2005)." For home-based exercises, providing participants with a manual of instructions and illustrations can improve adherence to the exercises prescribed (Madureira et al. 2007).

\section{Sustainability and Scalability Program attrition}

The median rate of attrition was $3 \%$ per month (range: 0-10\%) (Table 2). The study with the highest attrition rate reported losing 65 of the 198 participants for the following reasons: new or worsening health problems $(n=29)$, moving away $(n=6)$, participant choice $(n=12)$, and unknown $(n=12)$ (Steadman et al. 2003). Authors of one study reported its relatively low attrition rate was due to "telephone calls to those who failed to attend more than two training sessions, which provided active reinforcement to encourage participation in the program (Bunout et al. 2005)."

\section{Maintaining exercise after intervention}

Several studies stated that participants remained active after the intervention (Barnett et al. 2003; Li et al. 2005; Swanenburg et al. 2007). One Tai Chi program indicated that more than half the participants remained physically active after the intervention and authors described the potential for convincing participants to make an active lifestyle change: "Tai Chi appears to be an appealing exercise program, with benefits that go beyond functional balance improvements and falls reduction, and that can be regarded as a self-regulated, enjoyable, and socially engaging activity ( $\mathrm{Li}$ et al. 2004)."

\section{Resource implications}

The cost for exercise instruction varied based on whether the instruction was completed in a class setting versus one-on-one, the frequency and duration of supervised exercise, and the instructor qualifications. The estimated cost for instruction by a physical therapist of a successful program that provided weekly, one hour classes with 30 participants per class, supplemented with home exercises three times per week, was estimated at less than $\$ 2$ per participant per week (Madureira et al. 
2007). A second successful study had more frequent supervised exercise with three one-hour individual sessions provided per week at an outpatient research center (Protas et al. 2005). The estimated cost for this instruction is more than $\$ 100$ per participant per week.

\section{Discussion}

The USPSTF recommends exercise or physical therapy to prevent falls in community-dwelling adults age $\geq$ 65 years who are at increased risk for falls. Systematic reviews demonstrate that exercise programs are effective in reducing falls in community-dwelling elders (El-Khoury et al. 2013; Gillespie et al. 2012; Michael et al. 2010; Sherrington et al. 2011; Sherrington et al. 2008). Recent meta-analyses also examined whether different features of exercise programs are associated with greater falls prevention effects (Sherrington et al. 2011; Sherrington et al. 2008). While successful community-based fall prevention programs exist, additional efforts are needed to help integrate effective exercise programs with health care settings. This article builds on the previous work, using a complementary qualitative approach, and provides insight on how to implement exercise programs where participants are recruited from, or receive exercise in, a health care setting. As such, our findings are relevant to clinicians and health plan/integrated delivery system leaders who seek to strengthen their connections with exercise programs. Data from this review may be used to determine appropriate options for the types of exercise offered, location, frequency, duration, and type of supervision. In addition, we have synthesized information on approaches to maintaining adherence and reducing attrition, and have estimated some of the resource implications of program delivery.

Tinetti and Brach question whether all adults age $\geq 65$ or only those with an elevated risk for injury should be encouraged to participate (Tinetti et al. 2012). Although most fall prevention programs are designed for older adults with an elevated risk for falls and fall injury, Sherrington et al. (2011) recommended that programs should target both the general community and those at high risk for falls, and we found five studies that implemented successful interventions for older adults without an elevated risk (Buchner et al. 1997; Hornbrook et al. 1994; Kronhed et al. 2009; Lin et al. 2007; Robertson et al. 2001). One of these studies (Hornbrook et al. 1994) decreased the odds of falling by 0.85 among independently living $\mathrm{HMO}$ members age $\geq 65$. This program required limited resources for instruction and supervision by providing four initial weekly group meetings with up to 25 participants combined with exercises to follow at home. Quarterly maintenance sessions helped maintain participant motivation. To identify the targeted patient population, feasible screening strategies exist (Kenny et al. 2011;
Reuben et al. 2003), and can be implemented particularly effectively in settings with an electronic health record (Spears et al. 2013; Wenger et al. 2010) or care management system (Wenger et al. 2011). Potential participants may then be offered exercise programs after PCP review for appropriateness, much as participants in some of the studies we reviewed were recruited using PCP registries.

In regards to the type of exercise that should be included in a program, Sherrington et al. (2011) found that that exercise including a moderate or high challenge to balance was more effective in reducing falls than exercise lacking this component. Our study was not designed to confirm these findings directly, but noted that exercise programs in health care settings typically had multiple components (gait/balance/functional training and strength/resistance training being the most common); of five studies that were limited to a single exercise type, only one was successful.

To encourage patient participation, exercise programs should highlight a range of benefits in addition to fall prevention (Tinetti et al. 2012). Many studies have found benefits across a greater range of outcomes including quality of life (Ashburn et al. 2007a; Lin et al. 2007), participation in activities of daily living (Lin et al. 2007), physical functioning (Barnett et al. 2003; Bunout et al. 2005; Li et al. 2005; Madureira et al. 2007), and pain (Kronhed et al. 2009). Physicians and health plans should tout these benefits during referral to exercise programs because older adults are motivated to participate by a wide range of perceived benefits such as enjoyment, improved health, mood, and independence (Yardley et al. 2006), and some older people do not perceive a need for a fall prevention program when advertised as such (Calhoun et al. 2011). Of note, Sherrington et al. (2011) recommended that programs may be delivered in a group or home-based settings and we found that both home-based and classbased exercise can be successfully implemented; the decision about which programs to offer may depend on geography (e.g., urban versus rural), individual preferences, available resources, and patient needs for tailoring of exercises, more supervision, and/or socialization. While the costs for exercise instruction per participant are generally lower for class-based exercise, both classbased and home-based programs have other costs to consider. Class-based programs require space, and patients will bear cost of travel and time for transportation to the class, while home-based programs will bear the travel and time costs for transportation to patients' homes. Both programs may require equipment.

Implementers should also consider the trade-offs between patient adherence and program intensity. More frequent and longer duration exercise interventions are important for reducing fall rates, but may also decrease adherence and willingness to participate (Weerdesteyn 
2006). Programs will waste resources if participants do not exercise or drop out. Sherrington's meta-analysis suggested that the greatest effects on fall rates were from programs that included a minimum dose of $50 \mathrm{~h}$ of exercise (Sherrington et al. 2011; Sherrington et al. 2008). Achieving $50 \mathrm{~h}$ of exercise is possible through both a class such as a Tai Chi program, or a home-based option such as the Otago Exercise Program. Strategies for promoting adherence include regular phone calls, refresher sessions or home visits. Even with good adherence rates, programs will need to replenish their sample of participants on a regular basis due to attrition for health reasons.

Although existing studies elucidate the role that the health care setting may play in connecting older adults with exercise programs, several gaps remain. First, how the PCP should be involved in determining an older person's appropriateness for exercise, both initially and over time, has not been specified. Evidence shows that PCPs are important for identifying and referring patients to exercise programs and the Stopping Elderly Accidents, Deaths \& Injuries toolkit, developed by the Centers for Disease Control and Prevention, provides tools to help PCPs assess fall risk, educate patients, and select interventions (Centers for Disease Control and Prevention, 2015). Second, how exercise programs should be monitored to ensure that they meet minimum quality standards remains unknown (Tinetti et al. 2012). Third, it is unclear how exercise should be integrated with other fall prevention interventions. The Patient-Centered Outcomes Research Institute has funded a pragmatic multicenter trial of a fall prevention program (Trial Identifier: NCT02475850; www.stride-study.org) in which patients are being recruited from healthcare delivery systems; completion of this trial may address some of these knowledge gaps.

Our review has several limitations. First, we included only English-language studies. Second, we identified our source articles through existing systematic reviews of fall prevention and therefore may not have captured the most recent studies. Third, although we identified additional related papers to collect information on implementation, we are limited to what is reported in the studies. Therefore, we erred on the side of inclusion and provided findings even when they were based on a few studies. These limitations are mitigated by our detailed collection and synthesis of data from research that showed extensive variation in approach, thus increasing the likelihood that relevant themes were captured across a spectrum of programs germane to policymakers and implementers.

\section{Conclusions}

In conclusion, building on strong evidence that fall prevention programs focused on exercise can help prevent falls, we have provided more detailed strategies to help clinical and health system leaders integrate fall prevention programs with routine care. Such strategies, however, require that PCPs receive infrastructure support to identify patients who may benefit from an exercise program, and to refer patients to programs that meet clinical needs and patients' preferences.

\section{Abbreviations}

PCP, primary care provider; USPSTF, U.S. Preventive Services Task Force

\section{Competing interests}

The authors declare that they have no competing interests.

\section{Authors' contributions}

VS, ET, and DAG participated in the study concept and design, acquisition of data, analysis and interpretation of data, and preparation of manuscript. All authors read and approved the final manuscript.

\section{Acknowledgements}

The authors thank Debra Rose, PhD; Jon Pynoos, PhD; and Emily Nabors, MSG for their helpful comments on a previous version of this article.

\section{Funding}

This project was funded by a subcontract from the Fall Prevention Center of Excellence, funded by the Archstone Foundation. David Ganz was supported by a VA Career Development Award from the VA Health Services Research \& Development (HSR\&D) Service (Project \# VA CD2 08-012-1) through the VA Greater Los Angeles HSR\&D Center of Excellence. The Archstone Foundation and the U.S. Department of Veterans Affairs (VA) Greater Los Angeles Healthcare System had no role in the design, methods, subject recruitment, data collections, analysis and preparation of this paper.

\section{Consent for publication}

Not applicable.

Ethics approval and consent to participate

Not applicable.

\section{Author details}

${ }^{1}$ Pardee RAND Graduate School, RAND Corporation, 1776 Main St, Santa Monica, CA 90407, USA. ${ }^{2}$ Cedars-Sinai Medical Center, 8700 Beverly Blvd, Los Angeles, CA 90048, USA. ${ }^{3}$ VA Greater Los Angeles Healthcare System, David Geffen School of Medicine at UCLA, RAND Corporation, 11301 Wilshire Blvd., Building 158, Room 128, Los Angeles, CA 90073, USA.

Received: 7 March 2016 Accepted: 20 June 2016

Published online: 04 July 2016

\section{References}

Albanese A. Can falls be prevented in Parkinson's disease? J Neurol Neurosurg Psychiatry. 2007;78(7):661.

Ashburn A, Fazakarley L, Ballinger C, Pickering R, McLellan LD, Fitton C. A randomised controlled trial of a home based exercise programme to reduce the risk of falling among people with Parkinson's disease. J Neurol Neurosurg Psychiatry. 2007a;78(7):678-84

Ashburn A, Pickering RM, Fazakarley L, Ballinger C, McLellan DL, Fitton C. Recruitment to a clinical trial from the databases of specialists in Parkinson's disease. Parkinsonism Relat Disord. 2007b;13(1):35-9.

Ashburn A, Stack E, Ballinger C, Fazakarley L, Fitton C. The circumstances of falls among people with Parkinson's disease and the use of Falls Diaries to facilitate reporting. Disabil Rehabil. 2008;30(16):1205-12.

Barnett A, Smith B, Lord SR, Williams M, Baumand A. Community-based group exercise improves balance and reduces falls in at-risk older people: a randomised controlled trial. Age Ageing. 2003;32(4):407-14.

Beswick AD, Rees K, Dieppe P, Ayis S, Gooberman-Hill R, Horwood J, et al. Complex interventions to improve physical function and maintain independent living in elderly people: a systematic review and meta-analysis. Lancet. 2008:371(9614):725. 
Buchner D, Cress M, Wagner E, De Lateur B, Price R, Abrass I. The Seattle FICSIT/ Movelt study: the effect of exercise on gait and balance in older adults. J Am Geriatr Soc. 1993a;41(3):321.

Buchner D, Hornbrook M, Kutner N, Tinetti M, Ory M, Mulrow C, et al. Development of the common data base for the FICSIT trials. J Am Geriatr Soc. 1993b;41(3):297.

Buchner DM, Cress ME, de Lateur BJ, Esselman PC, Margherita AJ, Price R, et al. The effect of strength and endurance training on gait, balance, fall risk, and health services use in community-living older adults. J Gerontol A Biol Sci Med Sci. 1997;52(4):M218-24.

Bunout D, Barrera G, Avendano M, de la Maza P, Gattas V, Leiva L, et al. Results of a community-based weight-bearing resistance training programme for healthy Chilean elderly subjects. Age Ageing. 2005;34(1):80-3.

Calhoun R, Meischke H, Hammerback K, Bohl A, Poe P, Williams B, et al. Older adults' perceptions of clinical fall prevention programs: a qualitative study. J Aging Res. 2011;2011:867341.

Campbell AJ. Preventing fractures by preventing falls in older women. Can Med Assoc J. 2002;167(9):1005-6.

Campbell AJ, Robertson MC. Comprehensive Approach to Fall Prevention on a National Level: New Zealand. Clin Geriatr Med. 2010;26(4):719-31.

Campbell AJ, Robertson, M.C. Otago Exercise Programme to prevent falls in older adults. New Zealand: Otago Medical School, University of Otago; 2007.

Campbell AJ, Robertson MC, Gardner MM, Norton RN, Tilyard MW, Buchner DM. Randomised controlled trial of a general practice programme of home based exercise to prevent falls in elderly women. BMJ. 1997;315(7115):1065-9.

Campbell AJ, Robertson MC, Gardner MM, Norton RN, Buchner DM. Falls prevention over 2 years: a randomized controlled trial in women 80 years and older. Age Ageing. 1999a;28(6):513-8.

Campbell AJ, Robertson MC, Gardner MM, Norton RN, Buchner DM. Psychotropic medication withdrawal and a home-based exercise program to prevent falls: a randomized, controlled trial. J Am Geriatr Soc. 1999b;47(7):850-3.

Campbell AJ, Robertson MC, La Grow SJ, Kerse NM, Sanderson GF, Jacobs RJ, et al. Randomised controlled trial of prevention of falls in people aged $>$ or =75 with severe visual impairment: the VIP trial. BMJ. 2005;331(7520):817.

Carter ND, Khan KM, Petit MA, Heinonen A, Waterman C, Donaldson MG, et al. Results of a 10 week community based strength and balance training programme to reduce fall risk factors: a randomised controlled trial in 65-75 year old women with osteoporosis. Br J Sports Med. 2001;35(5):348-51.

Carter ND, Khan KM, McKay HA, Petit MA, Waterman C, Heinonen A, et al. Community-based exercise program reduces risk factors for falls in 65- to 75year-old women with osteoporosis: randomized controlled trial. CMAJ. 2002; 167(9):997-1004.

Centers for Disease Control and Prevention. Falls among older adults: an overview. 2016 [Available from: http://www.cdc.gov/homeandrecreationalsafety/falls/ adultfalls.html. Accessed March 2016.

Centers for Disease Control and Prevention. Stopping Elderly Accidents, Deaths, \& Injuries Tool Kit. 2015 [Available from: http://www.cdc.gov/steadi/index. html. Accessed March 2016].

Centre for Reviews and Dissemination. Systematic Reviews; University of York, 2009.

Clemson L, Singh MF, Bundy A, Cumming RG, Weissel E, Munro J, et al. LiFE Pilot Study: A randomised trial of balance and strength training embedded in daily life activity to reduce falls in older adults. Aust Occup Ther J. 2010;57(1):42-50.

Clemson L, Singh MAF, Bundy A, Cumming RG, Manollaras K, O'Loughlin P, et al. Integration of balance and strength training into daily life activity to reduce rate of falls in older people (the LiFE study): randomised parallel trial. BMJ. 2012;345:e4547

Dangour AD, Albala C, Aedo C, Elbourne D, Grundy E, Walker D, et al. A factorialdesign cluster randomised controlled trial investigating the cost-effectiveness of a nutrition supplement and an exercise programme on pneumonia incidence, walking capacity and body mass index in older people living in Santiago, Chile: the CENEX study protocol. Nutr J. 2007;6:14.

Dangour AD, Albala C, Allen E, Grundy E, Walker DG, Aedo C, et al. Effect of a nutrition supplement and physical activity program on pneumonia and walking capacity in Chilean older people: a factorial cluster randomized trial. PLoS Med. 2011;8(4):e1001023.

De Weerdt W, Feys $\mathrm{H}$. Assessment of physiotherapy for patients with stroke. Lancet. 2002;359(9302):182-3.

Dowswell G, Dowswell T, Lawler J, Green J, Young J. Patients' and caregivers' expectations and experiences of a physiotherapy intervention 1 year following stroke: a qualitative study. J Eval Clin Pract. 2002;8(3):361-5.
Ebrahim S, Thompson PW, Baskaran V, Evans K. Randomized placebo-controlled trial of brisk walking in the prevention of postmenopausal osteoporosis. Age Ageing. 1997;26(4):253-60.

El-Khoury F, Cassou B, Charles M-A, Dargent-Molina P. The effect of fall prevention exercise programmes on fall induced injuries in community dwelling older adults: systematic review and meta-analysis of randomised controlled trials. BMJ. 2013;347:f6234.

Freiberger E, Häberle L, Spirduso WW, Rixt Zijlstra G. Long-term effects of three multicomponent exercise interventions on physical performance and fall related psychological outcomes in community-dwelling older adults: a randomized controlled trial. J Am Geriatr Soc. 2012;60(3):437-46.

Freiberger E, Menz HB, Abu-Omar K, Rütten A. Preventing falls in physically active community-dwelling older people: a comparison of two intervention techniques. Gerontology. 2007;53(5):298-305

Ganz DA, Bao Y, Shekelle PG, Rubenstein LZ. Will my patient fall? JAMA. 2007; 297(1):77-86

Gardner MM, Buchner DM, Robertson MC, Campbell AJ. Practical implementation of an exercise-based falls prevention programme. Age Ageing. 2001;30(1):77-83.

Gates S, Fisher JD, Cooke M, Carter Y, Lamb SE. Multifactorial assessment and targeted intervention for preventing falls and injuries among older people in community and emergency care settings: systematic review and metaanalysis. BMJ. 2008;336(7636):130-3.

Gillespie LD, Robertson MC, Gillespie WJ, Sherrington C, Gates S, Clemson LM, et al. Interventions for preventing falls in older people living in the community. Cochrane Database Syst Rev. 2012;9:CD007146.

Green J, Forster A, Bogle S, Young J. Physiotherapy for patients with mobility problems more than 1 year after stroke: a randomised controlled trial. Lancet. 2002;359(9302):199-203.

Green J, Young J, Forster A, Collen F, Wade D. Combined analysis of two randomized trials of community physiotherapy for patients more than one year post stroke. Clin Rehabil. 2004;18(3):249-52.

Haines TP, Russell T, Brauer SG, Erwin S, Lane P, Urry S, et al. Effectiveness of a video-based exercise programme to reduce falls and improve health-related quality of life among older adults discharged from hospital: a pilot randomized controlled trial. Clin Rehabil. 2009;23(11):973-85.

Harmer P, Li F. Tai Chi and falls prevention in older people. Med Sport Sci. 2008; 52:124-134.

Hauer K, Rost B, Rutschle K, Opitz H, Specht N, Bartsch P, et al. Exercise training for rehabilitation and secondary prevention of falls in geriatric patients with a history of injurious falls. J Am Geriatr Soc. 2001;49(1):10-20.

Hornbrook MC, Stevens VJ, Wingfield DJ, Hollis JF, Greenlick MR, Ory MG. Preventing falls among community-dwelling older persons: results from a randomized trial. Gerontologist. 1994;34(1):16-23.

Iwamoto J, Suzuki H, Tanaka K, Kumakubo T, Hirabayashi H, Miyazaki Y, et al. Preventative effect of exercise against falls in the elderly: a randomized controlled trial. Osteoporos Int. 2009;20(7):1233-40.

Judge JO, Kenny A. Vitamin D and Quadriceps Exercise-Got Milk? Got a Chair? J Am Geriatr Soc. 2003;51(3):427-8.

Kenny R, Rubenstein L, Tinetti M, Brewer K, Cameron K, Capezuti E, et al. Summary of the updated american geriatrics society/british geriatrics society clinical practice guideline for prevention of falls in older persons. J Am Geriatr Soc. 2011;59(1):148-57.

Kronhed NG, Hallberg I, Odkvist L, Moller M. Effect of training on health-related quality of life, pain and falls in osteoporotic women. Adv Physiotherapy. 2009;11:242-6.

Lamb S, Becker C, Gillespie L, Smith J, Finnegan S, Potter R, et al. Reporting of complex interventions in clinical trials: development of a taxonomy to classify and describe fall-prevention interventions. Trials. 2011;12(1):125.

Latham NK, Anderson CS, Lee A, Bennett DA, Moseley A, Cameron ID. A randomized, controlled trial of quadriceps resistance exercise and vitamin $D$ in frail older people: the Frailty Interventions Trial in Elderly Subjects (FITNESS). J Am Geriatr Soc. 2003a;51(3):291-9.

Latham NK, Anderson CS, Reid IR. Effects of vitamin D supplementation on strength, physical performance, and falls in older persons: a systematic review. J Am Geriatr Soc. 2003b;51(9):1219-26.

Lehtola S, Koistinen P, Luukinen H. Falls and injurious falls late in home-dwelling life. Arch Gerontol Geriatr. 2006:42(2):217.

Li F, Harmer P, Fisher KJ, McAuley E. Tai Chi: improving functional balance and predicting subsequent falls in older persons. Med Sci Sports Exerc. 2004; 36(12):2046-52. 
Li F, Harmer P, Fisher KJ, McAuley E, Chaumeton N, Eckstrom E, et al. Tai Chi and fall reductions in older adults: a randomized controlled trial. J Gerontol A Biol Sci Med Sci. 2005;60(2):187-94.

Li F, Harmer P, Glasgow R, Mack KA, Sleet D, Fisher KJ, et al. Translation of an effective tai chi intervention into a community-based falls-prevention program. J Inf. 2008;98(7):1195-8.

Lin MR, Wolf SL, Hwang HF, Gong SY, Chen CY. A randomized, controlled trial of fall prevention programs and quality of life in older fallers. J Am Geriatr Soc. 2007;55(4):499-506.

Logghe $1 \mathrm{H}$, Zeeuwe PE, Verhagen AP, Wijnen-Sponselee RM, Willemsen SP, Bierma-Zeinstra SM, et al. Lack of effect of Tai Chi Chuan in preventing falls in elderly people living at home: a randomized clinical trial. J Am Geriatr Soc. 2009;57(1):70-5.

Logghe $\mathrm{HJ}$, Verhagen AP, Rademaker ACHJ, Zeeuwe PEM, Bierma-Zeinstra S, Van Rossum $E$, et al. Explaining the ineffectiveness of a Tai Chi fall prevention training for community-living older people: A process evaluation alongside a randomized clinical trial (RCT). Arch Gerontol Geriatr. 2011;52(3):357-62.

Luukinen H, Lehtola S, Jokelainen J, Vaananen-Sainio R, Lotvonen S, Koistinen P. Prevention of disability by exercise among the elderly: a population-based, randomized, controlled trial. Scand J Prim Health Care. 2006;24(4):199-205.

Luukinen $\mathrm{H}$, Lehtola S, Jokelainen J, Vaananen-Sainio R, Lotvonen S, Koistinen P. Pragmatic exercise-oriented prevention of falls among the elderly: a population-based, randomized, controlled trial. Prev Med. 2007:44(3):265-71.

Madureira MM, Takayama L, Gallinaro AL, Caparbo VF, Costa RA, Pereira RM. Balance training program is highly effective in improving functional status and reducing the risk of falls in elderly women with osteoporosis: a randomized controlled trial. Osteoporos Int. 2007;18(4):419-25.

Michael YL, Whitlock EP, Lin JS, Fu R, O'Connor EA, Gold R. Primary care-relevant interventions to prevent falling in older adults: a systematic evidence review for the US Preventive Services Task Force. Ann Intern Med. 2010;153(12):815.

Moyer VA. on behalf of the US Preventive Services Task Force. Prevention of Falls in Community-Dwelling Older Adults: US Preventive Services Task Force Recommendation Statement. Ann Intern Med Online. 2012;28.

Ory MG, Schechtman K, Miller JP, Hadley EC, Fiatarone MA, Province MA, et al. Frailty and injuries in later life: the FICSIT trials. J Am Geriatr Soc. 1993;41(3):283.

Protas EJ, Mitchell K, Williams A, Qureshy H, Caroline K, Lai EC. Gait and step training to reduce falls in Parkinson's disease. NeuroRehabilitation. 2005;20(3): 183-90.

Reuben DB, Roth C, Kamberg C, Wenger NS. Restructuring Primary Care Practices to Manage Geriatric Syndromes: The ACOVE-2 Intervention. J Am Geriatr Soc. 2003;51(12):1787-93.

Robertson MC, Campbell AJ. Optimisation of ACC's fall prevention programmes for older people. Univ Otago N Z. 2008;10(6):10.

Robertson MC, Devlin N, Gardner MM, Campbell AJ. Effectiveness and economic evaluation of a nurse delivered home exercise programme to prevent falls. 1: Randomised controlled trial. BMJ. 2001;322(7288):697-701.

Robertson MC, Campbell AJ, Gardner MM, Devlin N. Preventing injuries in older people by preventing falls: a meta-analysis of individual-level data. J Am Geriatr Soc. 2002;50(5):905-11.

Rubenstein LZ, Josephson KR. The epidemiology of falls and syncope. Clin Geriatr Med. 2002;18(2):141-58.

Rubenstein LZ, Josephson KR, Trueblood PR, Loy S, Harker JO, Pietruszka FM, et al. Effects of a group exercise program on strength, mobility, and falls among fall-prone elderly men. J Gerontol A Biol Sci Med Sci. 2000;55(6): M317-21.

Sherrington C, Whitney JC, Lord SR, Herbert RD, Cumming RG, Close JCT. Effective exercise for the prevention of falls: a systematic review and metaanalysis. J Am Geriatr Soc. 2008;56(12):2234-43.

Sherrington C, Tiedemann A, Fairhall N, Close JCT, Lord SR. Exercise to prevent falls in older adults: an updated meta-analysis and best practice recommendations. N S W Public Health Bull. 2011;22(4):78-83.

Spears GV, Roth CP, Miake-Lye IM, Saliba D, Shekelle PG, Ganz DA. Redesign of an electronic clinical reminder to prevent falls in older adults. Med Care. 2013; 51:\$37-43.

Steadman J, Donaldson N, Kalra L. A randomized controlled trial of an enhanced balance training program to improve mobility and reduce falls in elderly patients. J Am Geriatr Soc. 2003;51(6):847-52.

Stevens VJ HM, Wingfield DJ, Hollis JF, Greenlick MR. Recruitment and intervention for a falls prevention project: The study of accidential falls in the elderly (SAFE). Reducing frailty and falls in older persons. Sprinfield: Charles C Thomas; 1991. p. 277-92.
Stevens VJ, Hornbrook MC, Wingfield DJ, Hollis JF, Greenlick MR, Ory MG. Design and implementation of a falls prevention intervention for communitydwelling older persons. Behav Health Aging. 1992;2:57-73.

Stevens J, Corso P, Finkelstein E, Miller T. The costs of fatal and non-fatal falls among older adults. Injury Prev. 2006a;12(5):290-5.

Stevens J, Ryan G, Kresnow M. Fatalities and injuries from falls among older adults-United States, 1993-2003 and 2001-2005. MMWR. 2006b;55(45): $1222-4$.

Swanenburg J, de Bruin ED, Stauffacher M, Mulder T, Uebelhart D. Effects of exercise and nutrition on postural balance and risk of falling in elderly people with decreased bone mineral density: randomized controlled trial pilot study. Clin Rehabil. 2007;21(6):523-34.

Tinetti ME. Preventing falls in elderly persons. N Engl J Med. 2003;348(1):42-9.

Tinetti M, Brach J. Translating the fall prevention recommendations into a covered service: can it be done, and who should do it? Ann Intern Med. 2012;157(3):213.

U.S. Department of Labor. Occupational Employment and Wages, May 2015. Bureau of Labor Statistics; 2016 [Available from: http://www.bls.gov/oes/ home.htm. Accessed March 2016].

Walker DG, Aedo C, Albala C, Allen E, Dangour AD, Elbourne D, et al. Methods for economic evaluation of a factorial-design cluster randomised controlled trial of a nutrition supplement and an exercise programme among healthy older people living in Santiago, Chile: the CENEX study. BMC Health Serv Res. 2009; 9(1):85.

Weerdesteyn V, Rijken H, Geurts AC, Smits-Engelsman BC, Mulder T, Duysens J. A five-week exercise program can reduce falls and improve obstacle avoidance in the elderly. Gerontology. 2006;52(3):131-41.

Wenger NS, Roth CP, Hall WJ, Ganz DA, Snow V, Byrkit J, et al. Practice redesign to improve care for falls and urinary incontinence: primary care intervention for older patients. Arch Intern Med. 2010;170(19):1765.

Wenger NS, Roth CP, Martin D, Nickels L, Beckman R, Kamberg C, et al. Quality of care provided in a special needs plan using a nurse care manager model. J Am Geriatr Soc. 2011;59(10):1810-22.

Yardley L, Bishop FL, Beyer N, Hauer K, Kempen GIJM, Piot-Ziegler C, et al. Older people's views of falls-prevention interventions in six European countries. The Gerontologist. 2006;46(5):650-60.

Zeeuwe PEM, Verhagen AP, Bierma-Zeinstra SMA, Van Rossum E, Faber MJ, Koes BW. The effect of Tai Chi Chuan in reducing falls among elderly people: design of a randomized clinical trial in the Netherlands. BMC Geriatr. 2006;6(1):6.

\section{Submit your manuscript to a SpringerOpen ${ }^{\circ}$ journal and benefit from:}

- Convenient online submission

- Rigorous peer review

- Immediate publication on acceptance

- Open access: articles freely available online

- High visibility within the field

- Retaining the copyright to your article

Submit your next manuscript at springeropen.com 